\title{
Nonbinary Stabilizer Codes over Finite Fields
}

\author{
Avanti Ketkar*, Andreas Klappenecker†, Santosh Kumar* \\ Pradeep Kiran Sarvepalli \\ Texas A\&M University, Department of Computer Science, \\ College Station, TX 77843-3112
}

\begin{abstract}
One formidable difficulty in quantum communication and computation is to protect information-carrying quantum states against undesired interactions with the environment. In past years, many good quantum error-correcting codes had been derived as binary stabilizer codes. Fault-tolerant quantum computation prompted the study of nonbinary quantum codes, but the theory of such codes is not as advanced as that of binary quantum codes. This paper describes the basic theory of stabilizer codes over finite fields. The relation between stabilizer codes and general quantum codes is clarified by introducing a Galois theory for these objects. A characterization of nonbinary stabilizer codes over $\mathbf{F}_{q}$ in terms of classical codes over $\mathbf{F}_{q^{2}}$ is provided that generalizes the well-known notion of additive codes over $\mathbf{F}_{4}$ of the binary case. This paper derives lower and upper bounds on the minimum distance of stabilizer codes, gives several code constructions, and derives numerous families of stabilizer codes, including quantum Hamming codes, quadratic residue codes, quantum Melas codes, quantum $\mathrm{BCH}$ codes, and quantum character codes. The puncturing theory by Rains is generalized to additive codes that are not necessarily pure. Bounds on the maximal length of maximum distance separable stabilizer codes are given. A discussion of open problems concludes this paper.
\end{abstract}

This paper is dedicated to the memory of Professor Thomas Beth

\section{Introduction}

Reliable quantum information processing requires mechanisms to reduce the effects of environmental and operational noise. Fortunately, it is possible to alleviate the detrimental effects of decoherence by employing quantum errorcorrecting codes, so that one can engineer more reliable quantum communication schemes and quantum computers.

\footnotetext{
*Avanti Ketkar and Santosh Kumar are now with Microsoft Corporation, Seattle.

${ }^{\dagger}$ Contact author, e-mail: klappi at cs.tamu.edu
} 
The most widely studied class of quantum error-correcting codes are binary stabilizer codes, see $[7,8,14,18,20,27-30,33,37,39,40,42-46,48,54$, $55,57,68,80,89,91-94,96,99]$ and, in particular, the seminal works $[19,38]$. An appealing aspect of binary stabilizer codes is that there exist links to classical coding theory which ease the construction of good codes. More recently, some results were generalized to the case of nonbinary stabilizer codes $[1,3,4,16,23,24,34,35,41,47,49,56,64,70,79,83,85,86]$, but the theory is not nearly as complete as in the binary case.

We recall the basic principles of nonbinary stabilizer codes over finite fields in the next section. In Section 3, we introduce a Galois theory for quantum error-correcting codes. The original theory developed by Evariste Galois relates field extensions with groups. Oystein Ore distilled the essence of this correspondence and derived a significantly more general theory for pairs of lattices [74]. We use this framework and set up a Galois correspondence between quantum error-correcting codes and groups. This theory shows how some properties of general quantum codes, such as bounds on the minimum distance, can be deduced from results about stabilizer codes.

In Section 4, we recall that stabilizer codes over a finite field $\mathbf{F}_{q}$ correspond to additive codes over $\mathbf{F}_{q}$ that are self-orthogonal with respect to a trace-symplectic form [4]. We also establish the correspondence to additive codes over $\mathbf{F}_{q^{2}}$ that are self-orthogonal with respect to a trace-alternating form; remarkably, this basic construction had been missing in the literature, in spite of the fact that it is a generalization of the famous $\mathbf{F}_{4}$-codes [19].

The MacWilliams relations for weight enumerators of stabilizer codes are particularly easy to prove, as we show in Section 5. We then derive in Section 6 upper and lower bounds on the minimum distance of the best possible stabilizer codes. In Section 7 , we recall basic facts about cyclic stabilizer codes.

After laying the foundation in the first seven sections, we are able to construct numerous code families in the subsequent sections. In Section 8, we derive quantum Hamming codes; in Section 9, quantum quadratic residue codes; in Section 10, quantum Melas codes; and in Section 11, quantum BCH codes. In the latter case, we show that it is possible to extend quantum $\mathrm{BCH}$ codes. In Section 12, we generalize the known results about puncturing pure linear stabilizer codes to arbitrary additive codes, and we illustrate this theory by puncturing quantum BCH codes.

We show in Section 13 that stabilizer codes over $\mathbf{F}_{q}$ attaining the quantum Singleton bound cannot exceed a length of $q^{2}+1$, except in a few sporadic cases, assuming that the classical MDS conjecture holds. We give slightly weaker bounds for the length of MDS stabilizer codes without such 
an assumption. In Section 14, we derive an interesting class of quantum character codes. We give numerous code constructions in Section 15, and conclude the paper with a discussion of open questions.

We tried to keep the prerequisites to a minimum, so that readers from the coding theory community as well as from the quantum computing community can benefit. Apart from the basics of quantum computing, we recommend [19] and [40] for background on binary stabilizer codes, in addition to books on classical coding theory, such as [52] and [67]. The general theory of quantum codes is discussed in [61], and we assume that the reader is familiar with the notion of a detectable error, as introduced there. In general, we will omit proofs for results from our companion papers [2,84], but otherwise we tried to make this paper reasonably self-contained.

Notations. We assume throughout this paper that $\mathbf{F}_{q}$ denotes a finite field of characteristic $p$; in particular, $q$ always denotes a power of a prime $p$. The trace function from $\mathbf{F}_{q^{m}}$ to $\mathbf{F}_{q}$ is defined as $\operatorname{tr}_{q^{m} / q}(x)=\sum_{k=0}^{m-1} x^{q^{k}}$; we may omit the subscripts if $\mathbf{F}_{q}$ is the prime field. If $G$ is a group, then we denote by $Z(G)$ the center of this group. If $S \subseteq G$, then we denote by $C_{G}(S)$ the centralizer of $S$ in $G$. We write $H \leq G$ to express the fact that $H$ is a subgroup of $G$. The trace $\operatorname{Tr}(M)$ of a square matrix $M$ is the sum of the diagonal elements of $M$.

\section{Stabilizer Codes}

Let $q$ a power of a prime $p$, and let $\mathbf{C}^{q}$ be a $q$-dimensional complex vector space representing the states of a quantum mechanical system. We denote by $|x\rangle$ the vectors of a distinguished orthonormal basis of $\mathbf{C}^{q}$, where the labels $x$ range over the elements of a finite field $\mathbf{F}_{q}$ with $q$ elements. A quantum error-correcting code $Q$ is a $K$-dimensional subspace of $\mathbf{C}^{q^{n}}=\mathbf{C}^{q} \otimes \cdots \otimes \mathbf{C}^{q}$.

We need to select an appropriate error model so that we can measure the performance of a code. We simplify matters by choosing a basis $\mathcal{E}_{n}$ of the vector space of complex $q^{n} \times q^{n}$ matrices to represent a discrete set of errors. A stabilizer code is defined as the joint eigenspace of a subset of $\mathcal{E}_{n}$, so the error operators play a crucial role.

Error Bases. Let $a$ and $b$ be elements of the finite field $\mathbf{F}_{q}$. We define the unitary operators $X(a)$ and $Z(b)$ on $\mathbf{C}^{q}$ by

$$
X(a)|x\rangle=|x+a\rangle, \quad Z(b)|x\rangle=\omega^{\operatorname{tr}(b x)}|x\rangle,
$$


where $\operatorname{tr}$ denotes the trace operation from the extension field $\mathbf{F}_{q}$ to the prime field $\mathbf{F}_{p}$, and $\omega=\exp (2 \pi i / p)$ is a primitive $p$ th root of unity.

We form the set $\mathcal{E}=\left\{X(a) Z(b) \mid a, b \in \mathbf{F}_{q}\right\}$ of error operators. The set $\mathcal{E}$ has some interesting properties, namely (a) it contains the identity matrix, (b) the product of two matrices in $\mathcal{E}$ is a scalar multiple of another element in $\mathcal{E}$, and $(\mathrm{c})$ the trace $\operatorname{Tr}\left(A^{\dagger} B\right)=0$ for distinct elements $A, B$ of $\mathcal{E}$. A finite set of $q^{2}$ unitary matrices that satisfy the properties (a), (b), and (c) is called a nice error basis, see [60].

The set $\mathcal{E}$ of error operators forms a basis of the set of complex $q \times q$ matrices thanks to property (c). We include a proof that $\mathcal{E}$ is a nice error basis, because parts of our argument will be of independent interest in the subsequent sections.

Lemma 1. The set $\mathcal{E}=\left\{X(a) Z(b) \mid a, b \in \mathbf{F}_{q}\right\}$ is a nice error basis on $\mathbf{C}^{q}$.

Proof. The matrix $X(0) Z(0)$ is the identity matrix, so property (a) holds. We have $\omega^{\operatorname{tr}(b a)} X(a) Z(b)=Z(b) X(a)$, which implies that the product of two error operators is given by

$$
X(a) Z(b) X\left(a^{\prime}\right) Z\left(b^{\prime}\right)=\omega^{\operatorname{tr}\left(b a^{\prime}\right)} X\left(a+a^{\prime}\right) Z\left(b+b^{\prime}\right) .
$$

This is a scalar multiple of an operator in $\mathcal{E}$, hence property (b) holds.

Suppose that the error operators are of the form $A=X(a) Z(b)$ and $B=X(a) Z\left(b^{\prime}\right)$ for some $a, b, b^{\prime} \in \mathbf{F}_{q}$. Then

$$
\operatorname{Tr}\left(A^{\dagger} B\right)=\operatorname{Tr}\left(Z\left(b^{\prime}-b\right)\right)=\sum_{x \in \mathbf{F}_{q}} \omega^{\operatorname{tr}\left(\left(b^{\prime}-b\right) x\right)}
$$

The map $x \mapsto \omega^{\operatorname{tr}\left(\left(b^{\prime}-b\right) x\right)}$ is an additive character of $\mathbf{F}_{q}$. The sum of all character values is 0 unless the character is trivial; thus, $\operatorname{Tr}\left(A^{\dagger} B\right)=0$ when $b^{\prime} \neq b$.

On the other hand, if $A=X(a) Z(b)$ and $B=X\left(a^{\prime}\right) Z\left(b^{\prime}\right)$ are two error operators satisfying $a \neq a^{\prime}$, then the diagonal elements of the matrix $A^{\dagger} B=$ $Z(-b) X\left(a^{\prime}-a\right) Z\left(b^{\prime}\right)$ are 0 , which implies $\operatorname{Tr}\left(A^{\dagger} B\right)=0$. Thus, whenever $A$ and $B$ are distinct element of $\mathcal{E}$, then $\operatorname{Tr}\left(A^{\dagger} B\right)=0$, which proves (c).

Example 2. We give an explicit construction of a nice error basis with $q=4$ levels. The finite field $\mathbf{F}_{4}$ consists of the elements $\mathbf{F}_{4}=\{0,1, \alpha, \bar{\alpha}\}$. We denote the four standard basis vectors of the complex vector space $\mathbf{C}^{4}$ by $|0\rangle,|1\rangle,|\alpha\rangle$, and $|\bar{\alpha}\rangle$. Let $\mathbf{1}_{2}$ denote the $2 \times 2$ identity matrix, $\sigma_{x}=\left(\begin{array}{ll}0 & 1 \\ 1 & 0\end{array}\right)$, and $\sigma_{z}=\left(\begin{array}{lr}1 & 0 \\ 0 & -1\end{array}\right)$. Then

$$
\begin{aligned}
& X(0)=\mathbf{1}_{2} \otimes \mathbf{1}_{2}, \quad X(1)=\mathbf{1}_{2} \otimes \sigma_{x}, \quad X(\alpha)=\sigma_{x} \otimes \mathbf{1}_{2}, \quad X(\bar{\alpha})=\sigma_{x} \otimes \sigma_{x}, \\
& Z(0)=\mathbf{1}_{2} \otimes \mathbf{1}_{2}, \quad Z(1)=\sigma_{z} \otimes \mathbf{1}_{2}, \quad Z(\alpha)=\sigma_{z} \otimes \sigma_{z}, \quad Z(\bar{\alpha})=\mathbf{1}_{2} \otimes \sigma_{z} .
\end{aligned}
$$


We see that this nice error basis is obtained by tensoring the Pauli basis, a nice error basis on $\mathbf{C}^{2}$. The next lemma shows that this is a general design principle for nice error bases.

Lemma 3. If $\mathcal{E}_{1}$ and $\mathcal{E}_{2}$ are nice error bases, then

$$
\mathcal{E}=\left\{E_{1} \otimes E_{2} \mid E_{1} \in \mathcal{E}_{1}, E_{2} \in \mathcal{E}_{2}\right\}
$$

is a nice error basis as well.

The proof of this simple observation follows directly from the definitions.

Let $\mathbf{a}=\left(a_{1}, \ldots, a_{n}\right) \in \mathbf{F}_{q}^{n}$. We write $X(\mathbf{a})=X\left(a_{1}\right) \otimes \cdots \otimes X\left(a_{n}\right)$ and $Z(\mathbf{a})=Z\left(a_{1}\right) \otimes \cdots \otimes Z\left(a_{n}\right)$ for the tensor products of $n$ error operators. Our aim was to provide an error model that conveniently represents errors acting locally on one quantum system. Using the new notations, we can easily formulate this model.

Corollary 4. The set $\mathcal{E}_{n}=\left\{X(\mathbf{a}) Z(\mathbf{b}) \mid \mathbf{a}, \mathbf{b} \in \mathbf{F}_{q}^{n}\right\}$ is a nice error basis on the complex vector space $\mathbf{C}^{q^{n}}$.

Remark. Several authors have used an error basis that is equivalent to our definition of $\mathcal{E}_{n}$, see $[4,35,56,70]$. We have defined the operator $Z(b)$ in a slightly different way, so that the properties relevant for the design of stabilizer codes become more transparent. In particular, we can avoid an intermediate step that requires tensoring $p \times p$-matrices, and that allows us to obtain the trace-symplectic form directly, see Lemma 5 .

Stabilizer Codes. Let $G_{n}$ denote the group generated by the matrices of the nice error basis $\mathcal{E}_{n}$. It follows from equation (11) that

$$
G_{n}=\left\{\omega^{c} X(\mathbf{a}) Z(\mathbf{b}) \mid \mathbf{a}, \mathbf{b} \in \mathbf{F}_{q}^{n}, c \in \mathbf{F}_{p}\right\} .
$$

Note that $G_{n}$ is a finite group of order $p q^{2 n}$. We call $G_{n}$ the error group associated with the nice error basis $\mathcal{E}_{n}$.

A stabilizer code $Q$ is a non-zero subspace of $\mathbf{C}^{q^{n}}$ that satisfies

$$
Q=\bigcap_{E \in S}\left\{v \in \mathbf{C}^{q^{n}} \mid E v=v\right\}
$$

for some subgroup $S$ of $G_{n}$. In other words, $Q$ is the joint eigenspace to the eigenvalue 1 of a subgroup $S$ of the error group $G_{n}$.

Remark. A crucial property of a stabilizer code is that it contains all joint eigenvectors of $S$ with eigenvalue 1, as equation (2) indicates. If the code is smaller and does not exhaust all joint eigenvectors of $S$ with eigenvalue 1, then it is not a stabilizer code for $S$. 
Minimum Distance. The error correction and detection capabilities of a quantum error-correcting code $Q$ are the most crucial aspects of the code. Recall that a quantum code $Q$ is able to detect an error $E$ in the unitary group $U\left(q^{n}\right)$ if and only if the condition $\left\langle c_{1}|E| c_{2}\right\rangle=\lambda_{E}\left\langle c_{1} \mid c_{2}\right\rangle$ holds for all $c_{1}, c_{2} \in Q$, see [61].

It turns out that a stabilizer code $Q$ with stabilizer $S$ can detect all errors in $G_{n}$ that are scalar multiples of elements in $S$ or that do not commute with some element of $S$, see Lemma 11] In particular, an error in $G_{n}$ that is not detectable has to commute with all elements of the stabilizer. Commuting elements in $G_{n}$ are characterized as follows:

Lemma 5. Two elements $E=\omega^{c} X(\mathbf{a}) Z(\mathbf{b})$ and $E^{\prime}=\omega^{c^{\prime}} X\left(\mathbf{a}^{\prime}\right) Z\left(\mathbf{b}^{\prime}\right)$ of the error group $G_{n}$ satisfy the relation

$$
E E^{\prime}=\omega^{\operatorname{tr}\left(\mathbf{b} \cdot \mathbf{a}^{\prime}-\mathbf{b}^{\prime} \cdot \mathbf{a}\right)} E^{\prime} E .
$$

In particular, the elements $E$ and $E^{\prime}$ commute if and only if the trace symplectic form $\operatorname{tr}\left(\mathbf{b} \cdot \mathbf{a}^{\prime}-\mathbf{b}^{\prime} \cdot \mathbf{a}\right)$ vanishes.

Proof. It follows from equation (11) that $E E^{\prime}=\omega^{\operatorname{tr}\left(\mathbf{b} \cdot \mathbf{a}^{\prime}\right)} X\left(\mathbf{a}+\mathbf{a}^{\prime}\right) Z\left(\mathbf{b}+\mathbf{b}^{\prime}\right)$ and $E^{\prime} E=\omega^{\operatorname{tr}\left(\mathbf{b}^{\prime} \cdot \mathbf{a}\right)} X\left(\mathbf{a}+\mathbf{a}^{\prime}\right) Z\left(\mathbf{b}+\mathbf{b}^{\prime}\right)$. Therefore, multiplying $E^{\prime} E$ with the scalar $\omega^{\operatorname{tr}\left(\mathbf{b} \cdot \mathbf{a}^{\prime}-\mathbf{b}^{\prime} \cdot \mathbf{a}\right)}$ yields $E E^{\prime}$, as claimed.

We define the symplectic weight swt of a vector $(\mathbf{a} \mid \mathbf{b})$ in $\mathbf{F}_{q}^{2 n}$ as

$$
\operatorname{swt}((\mathbf{a} \mid \mathbf{b}))=\left|\left\{k \mid\left(a_{k}, b_{k}\right) \neq(0,0)\right\}\right| .
$$

The weight $\mathrm{w}(E)$ of an element $E=\omega^{c} X(\mathbf{a}) Z(\mathbf{b})$ in the error group $G_{n}$ is defined to be the number of nonidentity tensor components, $\mathrm{w}(E)=$ $\operatorname{swt}((\mathbf{a} \mid \mathbf{b}))$. In particular, the weight of a scalar multiple of the identity matrix is by definition zero.

A quantum code $Q$ has minimum distance $d$ if and only if it can detect all errors in $G_{n}$ of weight less than $d$, but cannot detect some error of weight $d$. We say that $Q$ is an $((n, K, d))_{q}$ code if and only if $Q$ is a $K$-dimensional subspace of $\mathbf{C}^{q^{n}}$ that has minimum distance $d$. An $\left(\left(n, q^{k}, d\right)\right)_{q}$ code is also called an $[[n, k, d]]_{q}$ code. We remark that some authors are more restrictive and use the bracket notation just to stabilizer codes.

We say that a quantum code $Q$ is pure to $t$ if and only if its stabilizer group $S$ does not contain non-scalar matrices of weight less than $t$. A quantum code is called pure if and only if it is pure to its minimum distance. As in $[19]$, we will always assume that an $[[n, 0, d]]_{q}$ code has to be pure.

Remark. (a) If a quantum error-correcting code can detect a set $\mathcal{D}$ of errors, then it can detect all errors in the linear span of $\mathcal{D}$. (b) A code of minimum distance $d$ can correct all errors of weight $t=\lfloor(d-1) / 2\rfloor$ or less. 


\section{Galois Connection}

We want to clarify the relation between stabilizer codes and more general quantum codes before we proceed further. Let us denote by $\mathcal{Q}$ the set of all subspaces of $\mathbf{C}^{q^{n}}$. The set $\mathcal{Q}$ is partially ordered by the inclusion relation. Any two elements of $\mathcal{Q}$ have a least upper bound and a greatest lower bound with respect to the inclusion relation, namely

$$
\sup \left\{Q, Q^{\prime}\right\}=Q+Q^{\prime} \text { and } \inf \left\{Q, Q^{\prime}\right\}=Q \cap Q^{\prime} .
$$

Therefore, $\mathcal{Q}$ is a complete (order) lattice. An element of this lattice is a quantum error-correcting code or is equal to the vector space $\{0\}$.

Let $\mathcal{G}$ denote the lattice of subgroups of the error group $G_{n}$. We will introduce two order-reversing maps between $\mathcal{G}$ and $\mathcal{Q}$ that establish a Galois connection. We will see that stabilizer codes are distinguished elements of $\mathcal{Q}$ that remain the same when mapped to the lattice $\mathcal{G}$ and back.

Let us define a map Fix from the lattice $\mathcal{G}$ of subgroups to the lattice $\mathcal{Q}$ of subspaces that associates to a group $S$ its joint eigenspace with eigenvalue 1 ,

$$
\operatorname{Fix}(S)=\bigcap_{E \in S}\left\{v \in \mathbf{C}^{q^{n}} \mid E v=v\right\} .
$$

We define for the reverse direction a map Stab from the lattice $\mathcal{Q}$ to the lattice $\mathcal{G}$ that associates to a quantum code $Q$ its stabilizer group $\operatorname{Stab}(Q)$,

$$
\operatorname{Stab}(Q)=\left\{E \in G_{n} \mid E v=v \text { for all } v \in Q\right\}
$$

We obtain four direct consequences of the definitions (31) and (44):

G1. If $Q_{1} \subseteq Q_{2}$ are subspaces of $\mathbf{C}^{q^{n}}$, then $\operatorname{Stab}\left(Q_{2}\right) \leq \operatorname{Stab}\left(Q_{1}\right)$.

G2. If $S_{1} \leq S_{2}$ are subgroups of $G_{n}$, then $\operatorname{Fix}\left(S_{2}\right) \leq \operatorname{Fix}\left(S_{1}\right)$.

G3. A subspace $Q$ of $\mathbf{C}^{q^{n}}$ satisfies $Q \subseteq \operatorname{Fix}(\operatorname{Stab}(Q))$.

G4. A subgroup $S$ of $G_{n}$ satisfies $S \leq \operatorname{Stab}(\operatorname{Fix}(S))$.

The first two properties establish that Fix and Stab are order-reversing maps. The extension properties G3 and G4 establish that Fix and Stab form a Galois connection, see [17, page 56]. The general theory of Galois connections establishes, among other results, that

$$
\operatorname{Fix}(S)=\operatorname{Fix}(\operatorname{Stab}(\operatorname{Fix}(S))) \quad \text { and } \quad \operatorname{Stab}(Q)=\operatorname{Stab}(\operatorname{Fix}(\operatorname{Stab}(Q)))
$$


holds for all $S$ in $\mathcal{G}$ and all $Q$ in $\mathcal{Q}$.

A subspace $Q$ of the vector space $\mathbf{C}^{q^{n}}$ satisfying G3 with equality is called a closed subspace, and a subgroup $S$ of the error group $G_{n}$ satisfying G4 with equality is called a closed subgroup. We record the main result of abstract Galois theory in the following proposition.

Proposition 6. The closed subspaces of the vector space $\mathbf{C}^{q^{n}}$ form a complete sublattice $\mathcal{Q}_{c}$ of the lattice $\mathcal{Q}$. The closed subgroups of $G_{n}$ form a complete sublattice $\mathcal{G}_{c}$ of the lattice $\mathcal{G}$ that is dual isomorphic to the lattice $\mathcal{Q}_{c}$.

Proof. This result holds for any Galois connection, see Theorem 10 in the book by Birkhoff [17, page 56].

We need to characterize the closed subspaces and subgroups to make this proposition useful. We begin with the closed subspaces because this is easier.

Lemma 7. A closed subspace is a stabilizer code or is 0-dimensional.

Proof. By definition, a closed subspace $Q$ satisfies

$$
Q=\operatorname{Fix}(\operatorname{Stab}(Q))=\bigcap_{E \in \operatorname{Stab}(Q)}\left\{v \in \mathbf{C}^{q^{n}} \mid E v=v\right\}
$$

hence is a stabilizer code or $\{0\}$.

Lemma 8. If $Q$ is a nonzero subspace of $\mathbf{C}^{q^{n}}$, then its stabilizer $S=$ $\operatorname{Stab}(Q)$ is an abelian group satisfying $S \cap Z\left(G_{n}\right)=\{1\}$.

Proof. Suppose that $E$ and $E^{\prime}$ are non-commuting elements of $S=\operatorname{Stab}(Q)$. By Lemma [5] we have $E E^{\prime}=\omega^{k} E^{\prime} E$ for some $\omega^{k} \neq 1$. A nonzero vector $v$ in $Q$ would have to satisfy $v=E E^{\prime} v=\omega^{k} E^{\prime} E v=\omega^{k} v$, contradiction. Therefore, $S$ is an abelian group. The stabilizer cannot contain any element $\omega^{k} \mathbf{1}$, unless $k=0$, which proves the second assertion.

Lemma 9. Suppose that $S$ is the stabilizer of a vector space $Q$. An orthogonal projector onto the joint eigenspace $\operatorname{Fix}(S)$ is given by

$$
P=\frac{1}{|S|} \sum_{E \in S} E
$$


Proof. A vector $v$ in $\operatorname{Fix}(S)$ satisfies $P v=v$, hence $\operatorname{Fix}(S)$ is contained in the image of $P$. Conversely, note that $E P=P$ holds for all $E$ in $S$, hence any vector in the image of $P$ is an eigenvector with eigenvalue 1 of all error operators $E$ in $S$. Therefore, $\operatorname{Fix}(S)=$ image $P$. The operator $P$ is idempotent, because

$$
P^{2}=\frac{1}{|S|} \sum_{E \in S} E P=\frac{1}{|S|} \sum_{E \in S} P=P
$$

holds. The inverse $E^{\dagger}$ of $E$ is contained in the group $S$, hence $P^{\dagger}=P$. Therefore, $P$ is an orthogonal projector onto $\operatorname{Fix}(S)$.

Remark. If $S$ is a nonabelian subgroup of the group $G_{n}$, then it necessarily contains the center $Z\left(G_{n}\right)$ of $G_{n}$; it follows that $P$ is equal to the all-zero matrix. Note that the image of $P$ has dimension $\operatorname{Tr}(P)=q^{n} /|S|$.

Lemma 10. A subgroup $S$ of $G_{n}$ is closed if and only if $S$ is an abelian subgroup that satisfies $S \cap Z\left(G_{n}\right)=\{1\}$ or if $S$ is equal to $G_{n}$.

Proof. Suppose that $S$ is a closed subgroup of $G_{n}$. The vector space $Q=$ $\operatorname{Fix}(S)$ is, by definition, either a stabilizer code or a 0 -dimensional vector space. We have $\operatorname{Stab}(\{0\})=G_{n}$. Furthermore, if $Q \neq\{0\}$, then $\operatorname{Stab}(Q)=$ $S$ is an abelian group satisfying $S \cap Z\left(G_{n}\right)=\{\mathbf{1}\}$, thanks to Lemma 8 .

Conversely, suppose that $S$ is an abelian subgroup of $G_{n}$ such that $S$ trivially intersects the center $Z\left(G_{n}\right)$. Let $S^{*}=\operatorname{Stab}(\operatorname{Fix}(S))$. We have $\operatorname{Fix}\left(S^{*}\right)=\operatorname{Fix}(\operatorname{Stab}(\operatorname{Fix}(S)))=\operatorname{Fix}(S)$, because this holds for any pair of maps that form a Galois connection. It follows from Lemma 9 that

$$
q^{n} /\left|S^{*}\right|=\operatorname{Tr}\left(\frac{1}{\left|S^{*}\right|} \sum_{E \in S^{*}} E\right)=\operatorname{Tr}\left(\frac{1}{|S|} \sum_{E \in S} E\right)=q^{n} /|S| .
$$

Since $S \leq S^{*}$, this shows that $S=S^{*}=\operatorname{Stab}(\operatorname{Fix}(S))$; hence, $S$ is a closed subgroup of $G_{n}$. We note that $\operatorname{Fix}\left(G_{n}\right)=\{0\}$, so that $G_{n}=\operatorname{Stab}\left(\operatorname{Fix}\left(G_{n}\right)\right)$ is closed.

The stabilizer codes are easier to study than arbitrary quantum codes, as we will see in the subsequent sections. If we know the error correction capabilities of stabilizer codes, then we get sometimes a lower bound on the minimum distance of an arbitrary code by the following simple observation:

Fact. An arbitrary quantum code $Q$ is contained in the larger stabilizer code $Q^{*}=\operatorname{Fix}(\operatorname{Stab}(Q))$. If an error $E$ can be detected by $Q^{*}$, then it can be detected by $Q$ as well. Therefore, if the stabilizer code $Q^{*}$ has minimum distance $d$, then the quantum code $Q$ has at least minimum distance $d$. 


\section{Additive Codes}

The previous section explored the relation between stabilizer codes and other quantum codes. We show next how stabilizer codes are related to classical codes (namely, additive codes over $\mathbf{F}_{q}$ or over $\mathbf{F}_{q^{2}}$ ). The classical codes allow us to characterize the errors in $G_{n}$ that are detectable by the stabilizer code.

If $S$ is a subgroup of $G_{n}$, then $C_{G_{n}}(S)$ denotes centralizer of $S$ in $G_{n}$,

$$
C_{G_{n}}(S)=\left\{E \in G_{n} \mid E F=F E \text { for all } F \in S\right\},
$$

and $S Z\left(G_{n}\right)$ denotes the group generated by $S$ and the center $Z\left(G_{n}\right)$. We first recall the following characterization of detectable errors (see also [4]; the interested reader can find a more general approach in $[58,59])$.

Lemma 11. Suppose that $S \leq G_{n}$ is the stabilizer group of a stabilizer code $Q$ of dimension $\operatorname{dim} Q>1$. An error $E$ in $G_{n}$ is detectable by the quantum code $Q$ if and only if either $E$ is an element of $S Z\left(G_{n}\right)$ or $E$ does not belong to the centralizer $C_{G_{n}}(S)$.

Proof. An element $E$ in $S Z\left(G_{n}\right)$ is a scalar multiple of a stabilizer; thus, it acts by multiplication with a scalar $\lambda_{E}$ on $Q$. It follows that $E$ is a detectable error.

Suppose now that $E$ is an error in $G_{n}$ that does not commute with some element $F$ of the stabilizer $S$; it follows that $E F=\lambda F E$ for some complex number $\lambda \neq 1$, see Lemma 5 . All vectors $u$ and $v$ in $Q$ satisfy the condition

$$
\langle u|E| v\rangle=\langle u|E F| v\rangle=\lambda\langle u|F E| v\rangle=\lambda\langle u|E| v\rangle
$$

hence, $\langle u|E| v\rangle=0$. It follows that the error $E$ is detectable.

Finally, suppose that $E$ is an element of $C_{G_{n}}(S) \backslash S Z\left(G_{n}\right)$. Seeking a contradiction, we assume that $E$ is detectable; this implies that there exists a complex scalar $\lambda_{E}$ such that $E v=\lambda_{E} v$ for all $v$ in $Q$. The scalar $\lambda_{E}$ cannot be zero, because $E$ commutes with the elements of $S$ so $E P=P E P=\lambda_{E} P$ and clearly $E P \neq 0$. Let $S^{*}$ denote the abelian group generated by $\lambda_{E}^{-1} E$ and by the elements of $S$. The joint eigenspace of $S^{*}$ with eigenvalue 1 has dimension $q^{n} /\left|S^{*}\right|<\operatorname{dim} Q=q^{n} /|S|$. This implies that not all vectors in $Q$ remain invariant under $\lambda_{E}^{-1} E$, in contradiction to the detectability of $E$.

Corollary 12. If a stabilizer code $Q$ has minimum distance $d$ and is pure to $t$, then all errors $E \in G_{n}$ with $1 \leq \operatorname{wt}(E)<\min \{t, d\}$ satisfy $\langle u|E| v\rangle=0$ for all $u$ and $v$ in $Q$. 
Proof. By assumption, the weight of $E$ is less than the minimum distance, so the error is detectable. However, $E$ is not an element of $Z\left(G_{n}\right) S$, since the code is pure to $t>\operatorname{wt}(E)$. Therefore, $E$ does not belong to $C_{G_{n}}(S)$, and the claim follows from equation (5).

Codes over $\mathbf{F}_{q}$. Lemma 11 characterizes the error detection capabilities of a stabilizer code with stabilizer group $S$ in terms of the groups $S Z\left(G_{n}\right)$ and $C_{G_{n}}(S)$. The phase information of an element in $G_{n}$ is not relevant for questions concerning the detectability, since an element $E$ of $G_{n}$ is detectable if and only if $\omega E$ is detectable. Thus, if we associate with an element $\omega^{c} X(\mathbf{a}) Z(\mathbf{b})$ of $G_{n}$ an element $(\mathbf{a} \mid \mathbf{b})$ of $\mathbf{F}_{q}^{2 n}$, then the group $S Z\left(G_{n}\right)$ is mapped to the additive code

$$
C=\left\{(\mathbf{a} \mid \mathbf{b}) \mid \omega^{c} X(\mathbf{a}) Z(\mathbf{b}) \in S Z\left(G_{n}\right)\right\}=S Z\left(G_{n}\right) / Z\left(G_{n}\right) .
$$

To describe the image of the centralizer, we need the notion of a tracesymplectic form of two vectors $(\mathbf{a} \mid \mathbf{b})$ and $\left(\mathbf{a}^{\prime} \mid \mathbf{b}^{\prime}\right)$ in $\mathbf{F}_{q}^{2 n}$,

$$
\left\langle(\mathbf{a} \mid \mathbf{b}) \mid\left(\mathbf{a}^{\prime} \mid \mathbf{b}^{\prime}\right)\right\rangle_{s}=\operatorname{tr}_{q / p}\left(\mathbf{b} \cdot \mathbf{a}^{\prime}-\mathbf{b}^{\prime} \cdot \mathbf{a}\right) .
$$

The centralizer $C_{G_{n}}(S)$ contains all elements of $G_{n}$ that commute with each element of $S$; thus, by Lemma $5 C_{G_{n}}(S)$ is mapped onto the tracesymplectic dual code $C^{\perp_{s}}$ of the code $C$,

$$
C^{\perp_{s}}=\left\{(\mathbf{a} \mid \mathbf{b}) \mid \omega^{c} X(\mathbf{a}) Z(\mathbf{b}) \in C_{G_{n}}(S)\right\} .
$$

The connection between these classical codes and the stabilizer code is made precise in the next theorem. This theorem is essentially contained in [4] and generalizes the well-known connection to symplectic codes $[19,38]$ of the binary case.

Theorem 13. An $((n, K, d))_{q}$ stabilizer code exists if and only if there exists an additive code $C \leq \mathbf{F}_{q}^{2 n}$ of size $|C|=q^{n} / K$ such that $C \leq C^{\perp_{s}}$ and $\operatorname{swt}\left(C^{\perp_{s}} \backslash C\right)=d$ if $K>1$ (and $\operatorname{swt}\left(C^{\perp_{s}}\right)=d$ if $K=1$ ).

Proof. Suppose that an $((n, K, d))_{q}$ stabilizer code $Q$ exists. This implies that there exists a closed subgroup $S$ of $G_{n}$ of order $|S|=q^{n} / K$ such that $Q=\operatorname{Fix}(S)$. The group $S$ is abelian and satisfies $S \cap Z\left(G_{n}\right)=1$, by Lemma 10. The quotient $C \cong S Z\left(G_{n}\right) / Z\left(G_{n}\right)$ is an additive subgroup of $\mathbf{F}_{q}^{2 n}$ such that $|C|=|S|=q^{n} / K$. We have $C^{\perp_{s}}=C_{G_{n}}(S) / Z\left(G_{n}\right)$ by Lemma 5 . Since $S$ is an abelian group, $S Z\left(G_{n}\right) \leq C_{G_{n}}(S)$, hence $C \leq C^{\perp_{s}}$. Recall that the weight of an element $\omega^{c} X(\mathbf{a}) Z(\mathbf{b})$ in $G_{n}$ is equal to $\operatorname{swt}(\mathbf{a} \mid \mathbf{b})$. 
If $K=1$, then $Q$ is a pure quantum code, thus $\operatorname{wt}\left(C_{G_{n}}(S)\right)=\operatorname{swt}\left(C^{\perp_{s}}\right)=d$. If $K>1$, then the elements of $C_{G_{n}}(S) \backslash S Z\left(G_{n}\right)$ have at least weight $d$ by Lemma 11] so that $\operatorname{swt}\left(C^{\perp_{s}} \backslash C\right)=d$.

Conversely, suppose that $C$ is an additive subcode of $\mathbf{F}_{q}^{2 n}$ such that $|C|=q^{n} / K, C \leq C^{\perp_{s}}$, and $\operatorname{swt}\left(C^{\perp_{s}} \backslash C\right)=d$ if $K>1$ (and $\operatorname{swt}\left(C^{\perp_{s}}\right)=d$ if $K=1$ ). Let

$$
N=\left\{\omega^{c} X(\mathbf{a}) Z(\mathbf{b}) \mid c \in \mathbf{F}_{p} \text { and }(\mathbf{a} \mid \mathbf{b}) \in C\right\} .
$$

Notice that $N$ is an abelian normal subgroup of $G_{n}$, because it is the preimage of $C=N / Z\left(G_{n}\right)$. Choose a character $\chi$ of $N$ such that $\chi\left(\omega^{c} \mathbf{1}\right)=\omega^{c}$. Then

$$
P_{N}=\frac{1}{|N|} \sum_{E \in N} \chi\left(E^{-1}\right) E
$$

is an orthogonal projector onto a vector space $Q$, because $P_{N}$ is an idempotent in the group ring $\mathbf{C}\left[G_{n}\right]$, see [58, Theorem 1]. We have

$$
\operatorname{dim} Q=\operatorname{Tr} P_{N}=\left|Z\left(G_{n}\right)\right| q^{n} /|N|=q^{n} /|C|=K .
$$

Each coset of $N$ modulo $Z\left(G_{n}\right)$ contains exactly one matrix $E$ such that $E v=v$ for all $v$ in $Q$. Set $S=\{E \in N \mid E v=v$ for all $v \in Q\}$. Then $S$ is an abelian subgroup of $G_{n}$ of order $|S|=|C|=q^{n} / K$. We have $Q=\operatorname{Fix}(S)$, because $Q$ is clearly a subspace of $\operatorname{Fix}(S)$, but $\operatorname{dim} Q=q^{n} /|S|=K$. An element $\omega^{c} X(\mathbf{a}) Z(\mathbf{b})$ in $C_{G_{n}}(S) \backslash S Z\left(G_{n}\right)$ cannot have weight less than $d$, because this would imply that $(\mathbf{a} \mid \mathbf{b}) \in C^{\perp_{s}} \backslash C$ has weight less than $d$, which is impossible. By the same token, if $K=1$, then all nonidentity elements of the centralizer $C_{G_{n}}(S)$ must have weight $d$ or higher. Therefore, $Q$ is an $((n, K, d))_{q}$ stabilizer code.

Codes over $\mathbf{F}_{q^{2}}$. A drawback of the codes in the previous paragraph is that the symplectic weight is somewhat unusual. In the binary case, reference [19] provided a remedy by relating binary stabilizer codes to additive codes over $\mathbf{F}_{4}$, allowing the use of the familiar Hamming weight. Somewhat surprisingly, the corresponding concept was not completely generalized to $\mathbf{F}_{q^{2}}$, although [56,70] and [79] paved the way to our approach. After circulating a first version of this manuscript, Gottesman drew our attention to another interesting approach that was initiated by Barnum, see $[12,13]$, where a sufficient condition for the existence of stabilizer codes is established using a symplectic form. 
Let $\left(\beta, \beta^{q}\right)$ denote a normal basis of $\mathbf{F}_{q^{2}}$ over $\mathbf{F}_{q}$. We define a tracealternating form of two vectors $v$ and $w$ in $\mathbf{F}_{q^{2}}^{n}$ by

$$
\langle v \mid w\rangle_{a}=\operatorname{tr}_{q / p}\left(\frac{v \cdot w^{q}-v^{q} \cdot w}{\beta^{2 q}-\beta^{2}}\right) .
$$

We note that the argument of the trace is invariant under the Galois automorphism $x \mapsto x^{q}$, so it is indeed an element of $\mathbf{F}_{q}$, which shows that (6) is well-defined.

The trace-alternating form is bi-additive, that is, $\langle u+v \mid w\rangle_{a}=\langle u \mid w\rangle_{a}+$ $\langle v \mid w\rangle_{a}$ and $\langle u \mid v+w\rangle_{a}=\langle u \mid v\rangle_{a}+\langle u \mid w\rangle_{a}$ holds for all $u, v, w \in \mathbf{F}_{q^{2}}^{n}$. It is $\mathbf{F}_{p^{-}}$ linear, but not $\mathbf{F}_{q}$-linear unless $q=p$. And it is alternating in the sense that $\langle u \mid u\rangle_{a}=0$ holds for all $u \in \mathbf{F}_{q^{2}}^{n}$. We write $u \perp_{a} w$ if and only if $\langle u \mid w\rangle_{a}=0$ holds.

We define a bijective map $\phi$ that takes an element $(\mathbf{a} \mid \mathbf{b})$ of the vector space $\mathbf{F}_{q}^{2 n}$ to a vector in $\mathbf{F}_{q^{2}}$ by setting $\phi((\mathbf{a} \mid \mathbf{b}))=\beta \mathbf{a}+\beta^{q} \mathbf{b}$. The map $\phi$ is isometric in the sense that the symplectic weight of $(\mathbf{a} \mid \mathbf{b})$ is equal to the Hamming weight of $\phi((\mathbf{a} \mid \mathbf{b}))$.

Lemma 14. Suppose that $c$ and $d$ are two vector of $\mathbf{F}_{q}^{2 n}$. Then

$$
\langle c \mid d\rangle_{s}=\langle\phi(c) \mid \phi(d)\rangle_{a}
$$

In particular, $c$ and $d$ are orthogonal with respect to the trace-symplectic form if and only if $\phi(c)$ and $\phi(d)$ are orthogonal with respect to the tracealternating form.

Proof. Let $c=(\mathbf{a} \mid \mathbf{b})$ and $d=\left(\mathbf{a}^{\prime} \mid \mathbf{b}^{\prime}\right)$. We calculate

$$
\begin{aligned}
& \phi(c) \cdot \phi(d)^{q}=\beta^{q+1} \mathbf{a} \cdot \mathbf{a}^{\prime}+\beta^{2} \mathbf{a} \cdot \mathbf{b}^{\prime}+\beta^{2 q} \mathbf{b} \cdot \mathbf{a}^{\prime}+\beta^{q+1} \mathbf{b} \cdot \mathbf{b}^{\prime} \\
& \phi(c)^{q} \cdot \phi(d)=\beta^{q+1} \mathbf{a} \cdot \mathbf{a}^{\prime}+\beta^{2 q} \mathbf{a} \cdot \mathbf{b}^{\prime}+\beta^{2} \mathbf{b} \cdot \mathbf{a}^{\prime}+\beta^{q+1} \mathbf{b} \cdot \mathbf{b}^{\prime}
\end{aligned}
$$

Therefore, the trace-alternating form of $\phi(c)$ and $\phi(d)$ is given by

$$
\langle\phi(c) \mid \phi(d)\rangle_{a}=\operatorname{tr}_{q / p}\left(\frac{\phi(c) \cdot \phi(d)^{q}-\phi(c)^{q} \cdot \phi(d)}{\beta^{2 q}-\beta^{2}}\right)=\operatorname{tr}_{q / p}\left(\mathbf{b} \cdot \mathbf{a}^{\prime}-\mathbf{a} \cdot \mathbf{b}^{\prime}\right),
$$

which is precisely the trace-symplectic form $\langle c \mid d\rangle_{s}$.

Theorem 15. An $((n, K, d))_{q}$ stabilizer code exists if and only if there exists an additive subcode $D$ of $\mathbf{F}_{q^{2}}^{n}$ of cardinality $|D|=q^{n} / K$ such that $D \leq D^{\perp_{a}}$ and $\operatorname{wt}\left(D^{\perp_{a}} \backslash D\right)=d$ if $K>1$ (and $\operatorname{wt}\left(D^{\perp_{a}}\right)=d$ if $K=1$ ). 
Proof. Theorem 13 shows that an $((n, K, d))_{q}$ stabilizer code exists if and only if there exists a code $C \leq \mathbf{F}_{q}^{2 n}$ with $|C|=q^{n} / K, C \leq C^{\perp_{s}}$, and $\operatorname{swt}\left(C^{\perp_{s}} \backslash C\right)=d$ if $K>1$ (and $\operatorname{swt}\left(C^{\perp_{s}}\right)=d$ if $K=1$ ). We obtain the statement of the theorem by applying the isometry $\phi$.

We obtain the following convenient condition for the existence of a stabilizer code as a direct consequence of the previous theorem.

Corollary 16. If there exists a classical $[n, k]_{q^{2}}$ additive code $D \leq \mathbf{F}_{q^{2}}$ such that $D \leq D^{\perp_{a}}$ and $d^{\perp_{a}}=\operatorname{wt}\left(D^{\perp_{a}}\right)$ then there exists an $\left[\left[n, n-2 k, \geq d^{\perp_{a}}\right]\right]_{q}$ stabilizer code that is pure to $d^{\perp_{a}}$.

Remark. It is not necessary to use a normal basis in the definition of the isometry $\phi$ and the trace-alternating form. Alternatively, we could have used a polynomial basis $(1, \gamma)$ of $\mathbf{F}_{q}^{2} / \mathbf{F}_{q}$. In that case, one can define the isometry $\phi$ by $\phi((\mathbf{a} \mid \mathbf{b}))=\mathbf{a}+\gamma \mathbf{b}$, and a compatible trace-alternating form by

$$
\langle v \mid w\rangle_{a^{\prime}}=\operatorname{tr}_{q / p}\left(\frac{v \cdot w^{q}-v^{q} \cdot w}{\gamma-\gamma^{q}}\right) .
$$

One can check that the statement of Lemma 14 is satisfied for this choice as well. Other variations on this theme are possible.

Classical codes. Self-orthogonal codes with respect to the trace-alternating form are not often studied in classical coding theory; more common are codes which are self-orthogonal with respect to a euclidean or hermitian inner product. We relate these concepts of orthogonality in this paragraph.

Consider the hermitian inner product $\mathbf{x}^{q} \cdot \mathbf{y}$ of two vectors $\mathbf{x}$ and $\mathbf{y}$ in $\mathbf{F}_{q^{2}}^{n}$; we write $\mathbf{x} \perp_{h} \mathbf{y}$ if and only if $\mathbf{x}^{q} \cdot \mathbf{y}=0$ holds.

Lemma 17. If two vectors $\mathbf{x}$ and $\mathbf{y}$ in $\mathbf{F}_{q^{2}}^{n}$ satisfy $\mathbf{x} \perp_{h} \mathbf{y}$, then they satisfy $\mathbf{x} \perp_{a} \mathbf{y}$. In particular, if $D \leq \mathbf{F}_{q^{2}}^{n}$, then $D^{\perp_{h}} \leq D^{\perp_{a}}$.

Proof. It follows from $\mathbf{x}^{q} \cdot \mathbf{y}=0$ that $\mathbf{x} \cdot \mathbf{y}^{q}=0$ holds, whence

$$
\langle\mathbf{x} \mid \mathbf{y}\rangle_{a}=\operatorname{tr}_{q / p}\left(\frac{\mathbf{x} \cdot \mathbf{y}^{q}-\mathbf{x}^{q} \cdot \mathbf{y}}{\beta^{2 q}-\beta^{2}}\right)=0,
$$

as claimed.

Therefore, any self-orthogonal code with respect to the hermitian inner product is self-orthogonal with respect to the trace-alternating form. In general, the two dual space $D^{\perp_{h}}$ and $D^{\perp_{a}}$ are not the same. However, if $D$ happens to be $\mathbf{F}_{q^{2}}$-linear, then the two dual spaces coincide. 
Lemma 18. Suppose that $D \leq \mathbf{F}_{q^{2}}^{n}$ is $\mathbf{F}_{q^{2}}$-linear, then $D^{\perp_{h}}=D^{\perp_{a}}$.

Proof. Let $q=p^{m}, p$ prime. If $D$ is a $k$-dimensional subspace of $\mathbf{F}_{q^{2}}^{n}$, then $D^{\perp_{h}}$ is a $(n-k)$-dimensional subspace of $\mathbf{F}_{q^{2}}^{n}$. We can also view $D$ as a $2 m k$-dimensional subspace of $\mathbf{F}_{p}^{2 m n}$, and $D^{\perp_{a}}$ as a $2 m(n-k)$-dimensional subspace of $\mathbf{F}_{p}^{2 m n}$. Since $D^{\perp_{h}} \subseteq D^{\perp_{a}}$ and the cardinalities of $D^{\perp_{a}}$ and $D^{\perp_{h}}$ are the same, we can conclude that $D^{\perp_{a}}=D^{\perp_{h}}$.

Corollary 19. If there exists an $\mathbf{F}_{q^{2}}$-linear $[n, k, d]_{q^{2}}$ code $B$ such that $B^{\perp_{h}} \leq B$, then there exists an $[[n, 2 k-n, \geq d]]_{q}$ quantum code that is pure to $d$.

Proof. The hermitian inner product is nondegenerate, so the hermitian dual of the code $D:=B^{\perp_{h}}$ is $B$. The $[n, n-k]_{q^{2}}$ code $D$ is $\mathbf{F}_{q^{2}}$-linear, so $D^{\perp_{h}}=D^{\perp_{a}}$ by Lemma 18, and the claim follows from Corollary 16]

So it suffices to consider hermitian forms in the case of $\mathbf{F}_{q^{2}}$-linear codes. We have to use the slightly more cumbersome trace-alternating form in the case of additive codes that are not linear over $\mathbf{F}_{q^{2}}$.

An elegant and surprisingly simple construction of quantum codes was introduced in 1996 by Calderbank and Shor [20] and by Steane [93]. The CSS code construction provides perhaps the most direct link to classical coding theory.

Lemma 20 (CSS Code Construction). Let $C_{1}$ and $C_{2}$ denote two classical linear codes with parameters $\left[n, k_{1}, d_{1}\right]_{q}$ and $\left[n, k_{2}, d_{2}\right]_{q}$ such that $C_{2}^{\perp} \leq$ $C_{1}$. Then there exists a $\left[\left[n, k_{1}+k_{2}-n, d\right]\right]_{q}$ stabilizer code with minimum distance $d=\min \left\{\operatorname{wt}(c) \mid c \in\left(C_{1} \backslash C_{2}^{\perp}\right) \cup\left(C_{2} \backslash C_{1}^{\perp}\right)\right\}$ that is pure to $\min \left\{d_{1}, d_{2}\right\}$.

Proof. Let $C=C_{1}^{\perp} \times C_{2}^{\perp} \leq \mathbf{F}_{q}^{2 n}$. If $\left(c_{1} \mid c_{2}\right)$ and $\left(c_{1}^{\prime} \mid c_{2}^{\prime}\right)$ are two elements of $C$, then we observe that

$$
\operatorname{tr}\left(c_{2} \cdot c_{1}^{\prime}-c_{2}^{\prime} \cdot c_{1}\right)=\operatorname{tr}(0-0)=0 .
$$

Therefore, $C \leq C^{\perp_{s}}$. Furthermore, the trace-symplectic dual of $C$ contains $C_{2} \times C_{1}$, and a dimensionality argument shows that $C^{\perp_{s}}=C_{2} \times C_{1}$. Since the cartesian product $C_{1}^{\perp} \times C_{2}^{\perp}$ has $q^{2 n-\left(k_{1}+k_{2}\right)}$ elements, the stabilizer code has dimension $q^{k_{1}+k_{2}-n}$ by Theorem 13. The claim about the minimum distance and purity of the code is obvious from the construction.

Corollary 21. If $C$ is a classical linear $[n, k, d]_{q}$ code containing its dual, $C^{\perp} \leq C$, then there exists an $[[n, 2 k-n, \geq d]]_{q}$ stabilizer code that is pure to $d$. 


\section{$5 \quad$ Weight Enumerators}

The Shor-Laflamme weight enumerators of an arbitrary $((n, K))_{q}$ quantum code $Q$ with orthogonal projector $P$ are defined by the polynomials

$$
\sum_{i=0}^{n} A_{i}^{\mathrm{SL}} z^{i}, \quad \text { with } \quad A_{i}^{\mathrm{SL}}=\frac{1}{K^{2}} \sum_{\substack{E \in G_{n} \\ \operatorname{wt}(E)=i}} \operatorname{Tr}\left(E^{\dagger} P\right) \operatorname{Tr}(E P),
$$

and

$$
\sum_{i=0}^{n} B_{i}^{\mathrm{SL}} z^{i}, \quad \text { with } \quad B_{i}^{\mathrm{SL}}=\frac{1}{K} \sum_{\substack{E \in G_{n} \\ \operatorname{wt}(E)=i}} \operatorname{Tr}\left(E^{\dagger} P E P\right),
$$

see [90] for the binary case. The weights $A_{i}^{\mathrm{SL}}$ and $B_{i}^{\mathrm{SL}}$ have a nice combinatorial interpretation in the case of stabilizer codes. Indeed, let $C \leq \mathbf{F}_{q}^{2 n}$ denote the additive code associated with the stabilizer code $Q$. Define the symplectic weights of $C$ and $C^{\perp_{s}}$ respectively by

$$
A_{i}=|\{c \in C \mid \operatorname{swt}(c)=i\}| \quad \text { and } \quad B_{i}=\left|\left\{c \in C^{\perp_{s}} \mid \operatorname{swt}(c)=i\right\}\right| .
$$

The next lemma belongs to the folklore of stabilizer codes.

Lemma 22. The Shor-Laflamme weights of an $((n, K))_{q}$ stabilizer code $Q$ are multiples of the symplectic weights of the associated additive codes $C$ and $C^{\perp_{s}}$; more precisely,

$$
A_{i}^{\mathrm{SL}}=p A_{i} \quad \text { and } \quad B_{i}^{\mathrm{SL}}=p B_{i} \quad \text { for } \quad 0 \leq i \leq n,
$$

where $p$ is the characteristic of the field $\mathbf{F}_{q}$.

Proof. Recall that

$$
P=\frac{1}{|S|} \sum_{E \in S} S
$$

for the stabilizer group $S$ of $Q$. The trace $\operatorname{Tr}(E P)$ is nonzero if and only if $E^{\dagger}$ is an element of $S Z\left(G_{n}\right)$. If $E^{\dagger} \in S Z\left(G_{n}\right)$, then $\operatorname{Tr}\left(E^{\dagger} P\right) \operatorname{Tr}(E P)=$ $\left(q^{n} /|S|\right)^{2}=K^{2}$. Therefore, $A_{i}^{\mathrm{SL}}$ counts the elements in $S Z\left(G_{n}\right)$ of weight $i$, so $A_{i}^{\mathrm{SL}}=\left|Z\left(G_{n}\right)\right| \times|\{c \in C \mid \operatorname{swt}(c)=i\}|=p A_{i}$.

If $E$ commutes with all elements in $S$, then $\operatorname{Tr}\left(E^{\dagger} P E P\right)=\operatorname{Tr}\left(P^{2}\right)=$ $\operatorname{Tr}(P)=K$. If $E$ does not commute with some element of $S$, then $E$ is detectable; more precisely, the proof of Lemma 11] shows that $P E P=0 P$, hence $\operatorname{Tr}\left(E^{\dagger} P E P\right)=0$. Therefore, $B_{i}^{\mathrm{SL}}$ counts the elements in $C_{G_{n}}(S)$ of weight $i$, hence $B_{i}^{\mathrm{SL}}=\left|Z\left(G_{n}\right)\right| \times\left|\left\{c \in C^{\perp_{s}} \mid \operatorname{swt}(c)=i\right\}\right|=p A_{i}^{\prime}$. 
Shor and Laflamme had been aware of the stabilizer case when they introduced their weight enumerators, so the combinatorial interpretation of the weights does not appear to be a coincidence. Recall that the Shor-Laflamme enumerators of arbitrary quantum codes are related by a MacWilliams identity, see $[77,90]$. For stabilizer codes, we can directly relate the symplectic weight enumerators of $C$ and $C^{\perp_{s}}$,

$$
A(z)=\sum_{i=0}^{n} A_{i} z^{i} \quad \text { and } \quad B(z)=\sum_{i=0}^{n} B_{i} z^{i},
$$

using a simple argument that is very much in the spirit of Jessie MacWilliams' original proof for euclidean dual codes [66].

Theorem 23. Let $C$ be an additive subcode of $\mathbf{F}_{q}^{2 n}$ with symplectic weight enumerator $A(z)$. Then the symplectic weight enumerator of $C^{\perp_{s}}$ is given by

$$
B(z)=\frac{\left(1+\left(q^{2}-1\right) z\right)^{n}}{|C|} A\left(\frac{1-z}{\left.1+\left(q^{2}-1\right) z\right)}\right) .
$$

Proof. Let $\chi$ be a nontrivial additive character of $\mathbf{F}_{p}$. We define for $b \in \mathbf{F}_{q}^{2 n}$ a character $\chi_{b}$ of the additive group $C$ by substituting the trace-symplectic form for the argument of the character $\chi$, such that

$$
\chi_{b}(c)=\chi\left(\langle c \mid b\rangle_{s}\right)
$$

The character $\chi_{b}$ is trivial if and only if $b$ is an element of $C^{\perp_{s}}$. Therefore, we obtain from the orthogonality relations of characters that

$$
\sum_{c \in C} \chi_{b}(c)= \begin{cases}|C| & \text { for } b \in C^{\perp_{s}} \\ 0 & \text { otherwise. }\end{cases}
$$

The following relation for polynomials is an immediate consequence

$$
\sum_{c \in C} \sum_{b \in \mathbf{F}_{q}^{2 n}} \chi_{b}(c) z^{\mathrm{swt}(b)}=\sum_{b \in \mathbf{F}_{q}^{2 n}} z^{\mathrm{swt}(b)} \sum_{c \in C} \chi_{b}(c)=|C| B(z) .
$$

The right hand side is a multiple of the weight enumerator of the code $C^{\perp_{s}}$. Let us have a closer look at the inner sum of the left-hand side. If we express the vector $c \in C$ in the form $c=\left(c_{1}, \ldots, c_{n} \mid d_{1}, \ldots, d_{n}\right)$, and expand 
the character and its trace-symplectic form, then we obtain

$$
\begin{aligned}
\sum_{b \in \mathbf{F}_{q}^{2 n}} \chi_{b}(c) z^{\operatorname{swt}(b)} & =\sum_{\left(a_{1}, \ldots, a_{n} \mid b_{1}, \ldots, b_{n}\right) \in \mathbf{F}_{q}^{2 n}} z^{\sum_{k=1}^{n} \operatorname{swt}\left(a_{k} \mid b_{k}\right)} \chi\left(\sum_{k=1}^{n} \operatorname{tr}\left(d_{k} a_{k}-b_{k} c_{k}\right)\right) \\
& =\sum_{\left(a_{1}, \ldots, a_{n} \mid b_{1}, \ldots, b_{n}\right) \in \mathbf{F}_{q}^{2 n}} \prod_{k=1}^{n} z^{\operatorname{swt}\left(a_{k} \mid b_{k}\right)} \chi\left(\operatorname{tr}\left(d_{k} a_{k}-b_{k} c_{k}\right)\right) \\
& =\prod_{k=1}^{n} \sum_{\left(a_{k} \mid b_{k}\right) \in \mathbf{F}_{q}^{2}} z^{\operatorname{swt}\left(a_{k} \mid b_{k}\right)} \chi\left(\operatorname{tr}\left(d_{k} a_{k}-b_{k} c_{k}\right)\right) .
\end{aligned}
$$

Recall that $\chi$ is a nontrivial character of $\mathbf{F}_{p}$, hence the map $\left(a_{k} \mid b_{k}\right) \mapsto$ $\chi\left(\operatorname{tr}\left(d_{k} a_{k}-b_{k} c_{k}\right)\right)$ is a nontrivial character of $\mathbf{F}_{q}^{2}$ for all $\left(c_{k} \mid d_{k}\right) \neq(0 \mid 0)$. Therefore, we can simplify the inner sum to

$$
\sum_{\left(a_{k} \mid b_{k}\right) \in \mathbf{F}_{q}^{2}} z^{\operatorname{swt}\left(a_{k} \mid b_{k}\right)} \chi\left(\operatorname{tr}\left(d_{k} a_{k}-b_{k} c_{k}\right)\right)= \begin{cases}1+\left(q^{2}-1\right) z & \text { if }\left(c_{k} \mid d_{k}\right)=(0,0), \\ 1-z & \text { if }\left(c_{k} \mid d_{k}\right) \neq(0,0) .\end{cases}
$$

It follows that

$$
\sum_{b \in \mathbf{F}_{q}^{2 n}} \chi_{b}(c) z^{\operatorname{swt}(b)}=(1-z)^{\operatorname{swt}(c)}\left(1+\left(q^{2}-1\right) z\right)^{n-\operatorname{swt}(c)} .
$$

Substituting this expression into equation (7), we find that

$$
\begin{aligned}
B(z) & =|C|^{-1} \sum_{c \in C} \sum_{b \in \mathbf{F}_{q}^{2 n}} \chi_{b}(c) z^{\mathrm{swt}(b)} \\
& =\frac{\left(1+\left(q^{2}-1\right) z\right)^{n}}{|C|} \sum_{c \in C}\left(\frac{1-z}{1+\left(q^{2}-1\right) z}\right)^{\operatorname{swt}(c)} \\
& =\frac{\left(1+\left(q^{2}-1\right) z\right)^{n}}{|C|} A\left(\frac{1-z}{1+\left(q^{2}-1\right) z}\right),
\end{aligned}
$$

which proves the claim.

The coefficient of $z^{j}$ in $\left(1+\left(q^{2}-1\right) z\right)^{n-x}(1-z)^{x}$ is given by the Krawtchouk polynomial of degree $j$ in the variable $x$,

$$
K_{j}(x)=\sum_{s=0}^{j}(-1)^{s}\left(q^{2}-1\right)^{j-s}\left(\begin{array}{c}
x \\
s
\end{array}\right)\left(\begin{array}{c}
n-x \\
j-s
\end{array}\right) .
$$


Corollary 24. Keeping the notation of the previous theorem, we have

$$
B_{j}=\frac{1}{|C|} \sum_{x=0}^{n} K_{j}(x) A_{x}
$$

Proof. According to the previous theorem, we have

$$
\begin{aligned}
B(z) & =\frac{\left(1+\left(q^{2}-1\right) z\right)^{n}}{|C|} A\left(\frac{1-z}{\left.1+\left(q^{2}-1\right) z\right)}\right) \\
& =\frac{1}{|C|} \sum_{x=0}^{n} A_{x}(1-z)^{x}\left(1+\left(q^{2}-1\right) z\right)^{n-x} .
\end{aligned}
$$

We obtain the result by comparing the coefficients of $z^{j}$ on both sides.

The theory of Shor-Laflamme weight enumerators [90] was considerably extended by Rains in $[77,78,81,82]$.

\section{Bounds}

We need some bounds on the achievable minimum distance of a quantum stabilizer code. The first theorem yields a bound that is well-suited for computer search.

Theorem 25. If an $((n, K, d))_{q}$ stabilizer code with $K>1$ exists, then there exists a solution to the optimization problem: minimize $\sum_{j=1}^{d-1} A_{j}$ subject to the constraints

1. $A_{0}=1$ and $A_{j} \geq 0$ for all $1 \leq j \leq n$;

2. $\sum_{j=0}^{n} A_{j}=q^{n} / K$;

3. $B_{j}=\frac{K}{q^{n}} \sum_{r=0}^{n} K_{j}(r) A_{r}$ holds for all $j$ in the range $0 \leq j \leq n$;

4. $A_{j}=B_{j}$ for all $j$ in $0 \leq j<d$ and $A_{j} \leq B_{j}$ for all $d \leq j \leq n$;

5. $(p-1)$ divides $A_{j}$ for all $j$ in the range $1 \leq j \leq n$.

Proof. If an $((n, K, d))_{q}$ stabilizer code exists, then the symplectic weight distribution of the associated additive code $C$ satisfies conditions 1) and 2). For each nonzero codeword $c$ in $C, \alpha c$ is again in $C$ for all $\alpha$ in $\mathbf{F}_{p}^{*}$, so 5) holds. Corollary 24 shows that 3 ) holds. Since the quantum code has minimum distance $d$, it follows that 4) holds. 
Remark 26. If we are interested in bounds for $\mathbf{F}_{q^{2}}$ linear codes, then we can replace condition 5 ) in the previous theorem by $q^{2}-1$ divides $A_{j}$. This will even help in characteristic 2 .

The next bound is more convenient when one wants to find bounds by hand. In particular, any function $f$ satisfying the constraints of the next theorem will yield a useful bound on the dimension of a stabilizer code. This approach was introduced by Delsarte for classical codes [31]. Binary versions of Theorem 27 and Corollary 28 were proved by Ashikhmin and Litsyn [5], see also [8].

Theorem 27. Let $Q$ be an $((n, K, d))_{q}$ stabilizer code of dimension $K>1$. Suppose that $S$ is a nonempty subset of $\{0, \ldots, d-1\}$ and $N=\{0, \ldots, n\}$. Let

$$
f(x)=\sum_{i=0}^{n} f_{i} K_{i}(x)
$$

be a polynomial satisfying the conditions

i) $f_{x}>0$ for all $x$ in $S$, and $f_{x} \geq 0$ otherwise;

ii) $f(x) \leq 0$ for all $x$ in $N \backslash S$.

Then

$$
K \leq \frac{1}{q^{n}} \max _{x \in S} \frac{f(x)}{f_{x}} .
$$

Proof. Suppose that $C \leq \mathbf{F}_{q}^{2 n}$ is the additive code associated with the stabilizer code $Q$. If we apply Corollary 24 to the trace-symplectic dual code $C^{\perp_{s}}$ of the code $C$, then we obtain

$$
A_{i}=\frac{1}{\left|C^{\perp_{s}}\right|} \sum_{x=0}^{n} K_{i}(x) B_{x} .
$$

Using this relation, we find that

$$
\begin{aligned}
\left|C^{\perp_{s}}\right| \sum_{i \in S} f_{i} A_{i} & \leq\left|C^{\perp_{s}}\right| \sum_{i=0}^{n} f_{i} A_{i} \\
& =\left|C^{\perp_{s}}\right| \sum_{i=0}^{n} f_{i}\left(\frac{1}{\left|C^{\perp_{s}}\right|} \sum_{x=0}^{n} K_{i}(x) B_{x}\right) \\
& =\sum_{x=0}^{n} B_{x} \sum_{i=0}^{n} f_{i} K_{i}(x) .
\end{aligned}
$$


By assumption, $f(x)=\sum_{i=0}^{n} f_{i} K_{i}(x)$; thus, we can simplify the latter inequality and obtain

$$
\left|C^{\perp_{s}}\right| \sum_{i \in S} f_{i} A_{i} \leq \sum_{x=0}^{n} B_{x} f(x) \leq \sum_{x \in S} B_{x} f(x)=\sum_{x \in S} A_{x} f(x),
$$

where the last equality follows from the fact that the stabilizer code has minimum distance $d$, meaning that $A_{x}=B_{x}$ holds for all $x$ in the range $0 \leq x<d$. We can conclude that

$$
\left|C^{\perp_{s}}\right| \leq\left(\sum_{x \in S} A_{x} f(x)\right) /\left(\sum_{x \in S} f_{x} A_{x}\right) \leq \max _{x \in S} \frac{f(x)}{f_{x}}
$$

which proves the theorem, since $\left|C^{\perp_{s}}\right|=q^{n} K$.

As an example, we demonstrate that the previous theorem implies the quantum Singleton bound. Linear programming yields in general better bounds, but for short lengths one can actually find codes meeting the quantum Singleton bound.

Corollary 28 (Quantum Singleton Bound). An $((n, K, d))_{q}$ stabilizer code with $K>1$ satisfies

$$
K \leq q^{n-2 d+2} .
$$

Proof. Let $S=\{0, \ldots, d-1\}$. If we choose the polynomial

$$
f(x)=q^{n-d+1} \prod_{j=d}^{n}\left(1-\frac{x}{j}\right),
$$

then $f(x)=0$ for all $x$ in $\{0, \ldots, n\} \backslash S$. We can express $f(x)$ in the form

$$
f(x)=q^{n-d+1}\left(\begin{array}{c}
n-x \\
n-d+1
\end{array}\right) /\left(\begin{array}{c}
n \\
n-d+1
\end{array}\right) .
$$

We can express this polynomial as $f(x)=\sum_{i=0}^{n} f_{i} K_{i}(x)$, where

$$
f_{i}=q^{-2 n} \sum_{x=0}^{n} f(x) K_{x}(i)=q^{1-d-n} \sum_{x=0}^{n} K_{x}(i)\left(\begin{array}{c}
n-x \\
n-d+1
\end{array}\right) /\left(\begin{array}{c}
n \\
n-d+1
\end{array}\right) .
$$

Notice that $\sum_{x=0}^{n} K_{x}(i)\left(\begin{array}{c}n-x \\ n-d+1\end{array}\right)=\left(\begin{array}{c}n-i \\ d-1\end{array}\right) q^{2(d-1)}$, see [63]; hence,

$$
f_{i}=q^{d-1-n}\left(\begin{array}{l}
n-i \\
d-1
\end{array}\right) /\left(\begin{array}{c}
n \\
n-d+1
\end{array}\right)>0 .
$$


We obtain for the fraction $r(x):=f(x) / f_{x}$ the value

$$
r(x)=\frac{f(x)}{f_{x}}=q^{2 n-2 d+2}\left(\begin{array}{c}
n-x \\
n-d+1
\end{array}\right) /\left(\begin{array}{l}
n-x \\
d-1
\end{array}\right) .
$$

An easy calculation shows that

$$
\frac{r(x)}{r(x+1)}=\frac{n-x-d+1}{d-x-1} .
$$

Seeking a contradiction, we assume that there exists an $((n, K, d))_{q}$ stabilizer code with $2 d \geq n+2$. In this case $r(x) / r(x+1) \leq 1$, so that $r(d-1)$ is the maximum of the values $r(x)$ with $x \in\{0, \ldots, d-1\}$. By Theorem [27] we have $K \leq r(d-1) / q^{n}=q^{n-2 d+2} /\left(\begin{array}{c}n-d+1 \\ d-1\end{array}\right)$. This yields a contradiction, since $\left(\begin{array}{c}n-d+1 \\ d-1\end{array}\right) K$ cannot be less than $q^{n-2 d+2} \leq 1$ for dimension $K>1$.

If $2 d<n+2$, then $r(x) / r(x+1)>1$, so $r(0)=f(0) / f_{0}$ is the largest among the values $r(x)$ with $x \in\{0, \ldots, d-1\}$. We have $r(0)=q^{2 n-2 d+2}$; whence, it follows from Theorem 27 that the dimension $K$ of the code is bounded by

$$
K \leq q^{-n} \max _{0 \leq x<d} \frac{f(x)}{f_{x}}=q^{n-2 d+2},
$$

which proves the claim.

The binary version of the quantum Singleton bound was first proved by Knill and Laflamme in [61], see also [5,8], and later generalized by Rains using weight enumerators in [79].

The quantum Hamming bound states that any pure $((n, K, d))_{q}$ stabilizer code satisfies

$$
\sum_{i=0}^{\lfloor(d-1) / 2\rfloor}\left(\begin{array}{l}
n \\
i
\end{array}\right)\left(q^{2}-1\right)^{i} \leq q^{n} / K,
$$

see $[36,38]$. Several researchers have tried to find impure stabilizer codes that beat the quantum Hamming bound. However, Gottesman has shown that impure single and double error-correcting binary quantum codes cannot beat the quantum Hamming bound [40]. In the same vein, Theorem 27 allows us to derive the Hamming bound for arbitrary stabilizer codes, at least when the minimum distance is small. We illustrate the method for single error-correcting codes, and note that the same approach works for double error-correcting codes as well.

Corollary 29 (Quantum Hamming Bound). An $((n, K, 3))_{q}$ stabilizer code with $K>1$ satisfies

$$
K \leq q^{n} /\left(n\left(q^{2}-1\right)+1\right) .
$$


Proof. Recall that the intersection number $p_{i j}^{k}$ of the Hamming association scheme $H\left(n, q^{2}\right)$ is the integer $p_{i j}^{k}=\left|\left\{z \in \mathbf{F}_{q^{2}}^{n} \mid d(x, z)=i, d(y, z)=j\right\}\right|$, where $x$ and $y$ are two vectors in $\mathbf{F}_{q}^{n}$ of Hamming distance $d(x, y)=k$. The intersection numbers are related to Krawtchouk polynomials by the expression

$$
p_{i j}^{k}=q^{-2 n} \sum_{u=0}^{n} K_{i}^{n}(u) K_{j}^{n}(u) K_{u}^{n}(k)
$$

see $[11]$.

After this preparation, we can proceed to derive the Hamming bound as a consequence of Theorem [27, Let

$$
f(x)=\sum_{j, k=0}^{1} \sum_{i=0}^{n} K_{j}^{n}(i) K_{k}^{n}(i) K_{i}^{n}(x)=q^{2 n}\left(p_{00}^{x}+p_{10}^{x}+p_{01}^{x}+p_{11}^{x}\right) .
$$

The triangle inequality implies that $p_{i j}^{k}=0$ if one of the three arguments exceeds the sum of the other two; hence, $f(x)=0$ for $x>2$. The coefficients of the Krawtchouk expansion $f(x)=\sum_{i=0}^{n} f_{i} K_{i}(x)$ obviously satisfy $f_{i}=$ $\left(K_{0}(i)+K_{1}(i)\right)^{2} \geq 0$. A straightforward calculation gives

$$
\begin{array}{ll}
f(0)=q^{2 n}\left(n\left(q^{2}-1\right)+1\right), & f_{0}=\left(n\left(q^{2}-1\right)+1\right)^{2}, \\
f(1)=q^{2 n+2}, & f_{1}=\left((n-1)\left(q^{2}-1\right)\right)^{2}, \\
f(2)=2 q^{2 n}, & f_{2}=\left((n-2)\left(q^{2}-1\right)-1\right)^{2} .
\end{array}
$$

It follows that

$$
\max \left\{f(0) / f_{0}, f(1) / f_{1}, f(2) / f_{2}\right\} \leq q^{2 n} /\left(n\left(q^{2}-1\right)+1\right)
$$

holds for all $n \geq 5$. Using Theorem 27] we obtain the claim for all $n \geq 5$. For the lengths $n<5$, we obtain the claim from the quantum Singleton bound.

One real disadvantage of Theorem 27 is that the number of terms increase with the minimum distance and this can lead to cumbersome calculations. However, one can derive more consequences from Theorem 27 see, for instance, $[5,8,63,71]$.

Lower Bounds. We conclude this section by giving the quantum version of the classical lower bounds by Gilbert and Varshamov. Basically, a simple counting argument is used to establish the existence of stabilizer codes.

Our first lemma generalizes an idea used by Gottesman in his proof of the binary case. 
Lemma 30. An $((n, K, \geq d))_{q}$ stabilizer code with $K>1$ exists provided that

$$
\left(q^{n} K-q^{n} / K\right) \sum_{j=1}^{d-1}\left(\begin{array}{l}
n \\
j
\end{array}\right)\left(q^{2}-1\right)^{j}<\left(q^{2 n}-1\right)(p-1)
$$

holds.

Proof. Let $L$ denote the multiset

$$
L=\left\{C^{\perp_{s}} \backslash C \mid C \leq C^{\perp_{s}} \leq \mathbf{F}_{q}^{2 n} \text { with }|C|=q^{n} / K\right\} .
$$

The elements of this multiset correspond to stabilizer codes of dimension $K$. Note that $L$ is nonempty, since there exists a code $C$ of size $q^{n} / K$ that is generated by elements of the form $(a \mid 0)$; the form of the generators ensures that $C \leq C^{\perp_{s}}$.

All nonzero vectors in $\mathbf{F}_{q}^{2 n}$ appear in the same number of sets in $L$. Indeed, the symplectic group $\operatorname{Sp}\left(2 n, \mathbf{F}_{q}\right)$ acts transitively on the set $\mathbf{F}_{q}^{2 n} \backslash\{0\}$, see [50, Proposition 3.2], which means that for any nonzero vectors $u$ and $v$ in $\mathbf{F}_{q}^{2 n}$ there exists $\tau \in \operatorname{Sp}\left(2 n, \mathbf{F}_{q}\right)$ such that $v=\tau u$. Therefore, $u$ is contained in $C^{\perp_{s}} \backslash C$ if and only if $v$ is contained in the element $(\tau C)^{\perp_{s}} \backslash \tau C$ of $L$.

The transitivity argument shows that any nonzero vector in $\mathbf{F}_{q}^{2 n}$ occurs in $|L|\left(q^{n} K-q^{n} / K\right) /\left(q^{2 n}-1\right)$ elements of $L$. Furthermore, a nonzero vector and its $\mathbf{F}_{p}^{\times}$-multiples are contained in the exact same sets of $L$. Thus, if we delete all sets from $L$ that contain a nonzero vector with symplectic weight less than $d$, then we remove at most

$$
\frac{\sum_{j=1}^{d-1}\left(\begin{array}{c}
n \\
j
\end{array}\right)\left(q^{2}-1\right)^{j}}{p-1}|L| \frac{\left(q^{n} K-q^{n} / K\right)}{q^{2 n}-1}
$$

sets from $L$. By assumption, this number is less than $|L|$; hence, there exists an $((n, K, \geq d))_{q}$ stabilizer code.

The Gilbert-Varshamov bound provides surprisingly good codes, even for smaller lengths, when the characteristic of the field is not too small. If $n \equiv k \bmod 2$, then we can significantly strengthen the bound.

Lemma 31. If $k \geq 1, n \equiv k \bmod 2$ and

$$
\left(q^{n+k}-q^{n-k}\right) \sum_{j=1}^{d-1}\left(\begin{array}{l}
n \\
j
\end{array}\right)\left(q^{2}-1\right)^{j-1}<\left(q^{2 n}-1\right)
$$

holds, then there exists an $\mathbf{F}_{q^{2}}$-linear $[[n, k, d]]_{q}$ stabilizer code. 
Proof. The proof is almost the same as in the previous lemma, except that we list only codes $C$ that are linear, meaning that $\phi(C)$ is a vector space over $\mathbf{F}_{q^{2}}$. We repeat the previous argument with the multiset

$$
L=\left\{C^{\perp_{s}} \backslash C\left|C \leq C^{\perp_{s}} \leq F_{q}^{n},\right| C \mid=q^{n-k}, \phi(C) \text { is } \mathbf{F}_{q^{2}} \text {-linear }\right\} .
$$

Note that each set $C^{\perp_{s}} \backslash C$ in $L$ contains now all $\mathbf{F}_{q^{2}}^{\times}$-multiples of a nonzero vector, not just the $\mathbf{F}_{p}^{\times}$-multiples, which proves the statement.

Feng and Ma have recently shown that one can extend the previous result to even prove the existence of pure stabilizer codes, but much more delicate counting arguments are needed in that case, see [36]. We are not aware of short proofs for this stronger result.

The previous lemma allows us to derive good quantum codes, especially for larger alphabets. We illustrate this fact by proving the existence of MDS stabilizer codes, see Section 13 for more details on such codes.

Corollary 32. If $2 \leq d \leq\lceil n / 2\rceil$ and $q^{2}-1 \geq\left(\begin{array}{l}n \\ d\end{array}\right)$, then there exists a linear $[[n, n-2 d+2, d]]_{q}$ stabilizer code.

Proof. The assumption $d \leq\lceil n / 2\rceil$ implies that $\left(\begin{array}{l}n \\ 1\end{array}\right) \leq\left(\begin{array}{l}n \\ 2\end{array}\right) \leq \cdots \leq\left(\begin{array}{l}n \\ d\end{array}\right)$, so the maximum value of these binomial coefficients is at most $q^{2}-1$. Let $k=n-2 d+2$. It follows from the assumption that $k \geq 1$ and $n \equiv k \bmod 2$. It remains to show that (9) holds. For the choice $k=n-2 d+2$, the left hand side of (9) equals

$$
\begin{gathered}
\left(q^{2 n-2 d+2}-q^{2 d-2}\right) \sum_{j=1}^{d-1}\left(\begin{array}{c}
n \\
j
\end{array}\right) \\
\left(q^{2}-1\right)^{j-1} \leq\left(q^{2 n-2 d+2}-q^{2 d-2}\right) \sum_{j=1}^{d-1}\left(q^{2}-1\right)^{j} \\
=\left(q^{2 n-2 d+2}-q^{2 d-2}\right) \frac{\left(q^{2}-1\right)^{d}-\left(q^{2}-1\right)}{q^{2}-2} .
\end{gathered}
$$

We claim that the latter term is less than $q^{2 n}-1$. To prove this, it suffices to show that

$$
q^{2 n-2 d+2} \frac{\left(q^{2}-1\right)^{d}-\left(q^{2}-1\right)}{q^{2}-2} \leq q^{2 n}
$$

holds. The latter inequality is equivalent to $\left(q^{2}-1\right)^{d} \leq q^{2 d}-2 q^{2 d-2}+q^{2}-1$, and it is not hard to see that this inequality holds. Indeed, note that

$$
q^{2 d}=\left(\left(q^{2}-1\right)+1\right)^{d}=\left(q^{2}-1\right)^{d}+\sum_{j=0}^{d-1}\left(\begin{array}{l}
d \\
j
\end{array}\right)\left(q^{2}-1\right)^{j} .
$$


Recall that $\left(\begin{array}{l}d \\ j\end{array}\right)=\left(\begin{array}{c}d-1 \\ j-1\end{array}\right)+\left(\begin{array}{c}d-1 \\ j\end{array}\right) ;$ hence,

$$
\begin{aligned}
q^{2 d}-2 q^{2 d-2}-\left(q^{2}-1\right)^{d} & =\sum_{j=0}^{d-1}\left(\left(\begin{array}{l}
d \\
j
\end{array}\right)-2\left(\begin{array}{c}
d-1 \\
j
\end{array}\right)\right)\left(q^{2}-1\right)^{j}, \\
& =\sum_{j=0}^{d-1}(\underbrace{\left(\begin{array}{c}
d-1 \\
j-1
\end{array}\right)-\left(\begin{array}{c}
d-1 \\
j
\end{array}\right)}_{\alpha(j):=})\left(q^{2}-1\right)^{j} .
\end{aligned}
$$

We have $\alpha(j)=-\alpha(d-j)$ for $0 \leq j \leq d-1$, and $\alpha(j) \geq 0$ for $j \geq d / 2$. This shows that all negative terms get canceled by larger positive terms and we can conclude that $q^{2 d}-2 q^{2 d-2}-\left(q^{2}-1\right)^{d} \geq 0$ for $d \geq 2$; this implies inequality (10) and consequently shows that (9) holds.

Example 33. Recall that there does not exist a $[[7,1,4]]_{2}$ code, see [19]. In contrast, the existence of a $[[7,1,4]]_{q}$ code for all prime powers $q \geq 7$ is guaranteed by the preceding corollary. It also shows that there exist $[[6,2,3]]_{q}$ for all prime powers $q \geq 5$ and $[[7,3,3]]_{q}$ for all prime powers $q \geq 7$, which slightly generalizes [34].

\section{Cyclic Codes}

We shall now restrict our attention to linear quantum codes and derive several families of quantum codes from classical linear codes. In essence we make use of the hermitian and CSS constructions (Lemmas 19-21). Hence, we need to look for classical codes that are self-orthogonal with respect to the hermitian or the euclidean product or families of nested codes like the $\mathrm{BCH}$ codes.

In case of cyclic codes identifying the self-orthogonal codes can be translated into equivalent conditions on the generator polynomial of the code or its defining set. Let $\sigma$ denote the automorphism of the field $\mathbf{F}_{q^{2}}$ given by $\sigma(x)=x^{q}$. We can define an action of $\sigma$ on the polynomial ring $\mathbf{F}_{q^{2}}[x]$ by

$$
h(x)=\sum_{k=0}^{n} h_{k} x^{k} \longmapsto h^{\sigma}(x)=\sum_{k=0}^{n} \sigma\left(h_{k}\right) x^{k} .
$$

Lemma 34. Suppose that $B$ is a classical cyclic $[n, k, d]_{q^{2}}$ code with generator polynomial $g(x)$ and check polynomial $h(x)=\left(x^{n}-1\right) / g(x)$. If $g(x) d i$ vides $\sigma\left(h_{0}\right)^{-1} x^{k} h^{\sigma}(1 / x)$, then $B^{\perp_{h}} \subseteq B$, and there exists an $[[n, 2 k-n, \geq d]]_{q}$ stabilizer code that is pure to $d$. 
Proof. If $h(x)$ is the check polynomial of $B$, then $h^{\sigma}(x)$ is the check polynomial of $\sigma(B)$. The generator polynomial of the dual code $\sigma(B)^{\perp}=B^{\perp_{h}}$ is given by $\sigma\left(h_{0}\right)^{-1} x^{k} h^{\sigma}(1 / x)$, the normalized reciprocal polynomial of $h^{\sigma}(x)$. Therefore, the condition that the polynomial $g(x)$ divides $\sigma\left(h_{0}\right)^{-1} x^{k} h^{\sigma}(1 / x)$ is equivalent to the condition $B^{\perp_{h}} \subseteq B$. The stabilizer code follows from Corollary [19.

The polynomial $x^{n}-1$ of $\mathbf{F}_{q^{2}}[x]$ has simple roots if and only if $n$ and $q$ are coprime. If the latter condition is satisfied, then there exists a positive integer $m$ such that the field $\mathbf{F}_{q^{2 m}}$ contains a primitive $n$th root of unity $\beta$. In that case, one can describe a cyclic code with generator polynomial $g(x)$ in terms of its defining set $Z=\left\{k \mid g\left(\beta^{k}\right)=0\right.$ for $\left.0 \leq k<n\right\}$. The following Lemma summarizes various equivalent conditions on self-orthogonal codes in terms of the generator polynomial and the defining set $Z$.

Lemma 35. Let $\operatorname{gcd}\left(n, q^{2}\right)=1$ and $C$ be a classical cyclic $[n, k, d]_{q^{2}}$ code whose generator polynomial is $g(x)$ and defining set $Z$. If any of the following equivalent conditions are satisfied

(i) $x^{n}-1 \equiv 0 \bmod g(x) g^{*}(x)$ where $g^{*}(x)=x^{n-k} g^{\sigma}(1 / x)$

(ii) $Z \subseteq\{-q z \mid z \in N \backslash Z\}$

(iii) $Z \cap Z^{-q}=\emptyset$, where $Z^{-q}=\{-q z \mid z \in Z\}$

then $C^{\perp_{h}} \subseteq C$ and there exists an $[[n, 2 k-n, \geq d]]_{q}$ stabilizer code that is pure to $d$.

Proof. Let $h(x)=\left(x^{n}-1\right) / g(x)$ be the check polynomial of $C$. Then $h^{\sigma}(x)=$ $\sigma\left(\left(x^{n}-1\right) / g(x)\right)=\left(x^{n}-1\right) / g^{\sigma}(x)$. From Lemma 34 we know that $C$ contains its hermitian dual if $\mathrm{g}(\mathrm{x})$ divides $\sigma\left(h_{0}\right)^{-1} x^{k} h^{\sigma}(1 / x)$ viz. $g(x) \mid \sigma\left(h_{0}\right)^{-1}(1-$ $\left.x^{n}\right) /\left(x^{n-k} g^{\sigma}(1 / x)\right)$, which implies $x^{n}-1 \equiv 0 \bmod g(x) g^{*}(x)$ which proves (i).

The generator polynomial $g(x)$ of $C$ is given by $g(x)=\prod_{z \in Z}\left(x-\beta^{z}\right)$, hence its check polynomial is of the form

$$
h(x)=\left(x^{n}-1\right) / g(x)=\prod_{z \in N \backslash Z}\left(x-\beta^{z}\right) .
$$

Applying the automorphism $\sigma$ yields $h^{\sigma}(x)=\prod_{z \in N \backslash Z}\left(x-\beta^{q z}\right)$. Therefore, the generator polynomial of $C^{\perp_{h}}$ is given by

$$
\begin{aligned}
h^{\sigma}(0)^{-1} x^{k} h^{\sigma}(1 / x) & =h^{\sigma}(0)^{-1} \prod_{z \in N \backslash Z}\left(1-\beta^{q z} x\right) \\
& =\prod_{z \in N \backslash Z}\left(x-\beta^{-q z}\right) ;
\end{aligned}
$$

in the last equality, we have used the fact that $h^{\sigma}(0)^{-1}=\prod_{z \in N \backslash Z}\left(-\beta^{-q z}\right)$. By Lemma 34 $B^{\perp_{h}} \subseteq B$ if and only if the generator polynomial $g(x)$ divides 
$h^{\sigma}(0)^{-1} x^{k} h^{\sigma}(1 / x)$. The latter condition is equivalent to the fact that $Z$ is a subset of $\{-q z \mid z \in N \backslash Z\}$ and (ii) follows. From (ii) we know that $C^{\perp_{h}} \subseteq C$ if and only if $Z \subseteq\{-q z \mid z \in N \backslash Z\}$. In other words $Z^{-q} \subseteq N \backslash Z$. Hence $Z \cap Z^{-q}=\emptyset$. An $[[n, 2 k-n, \geq d]]_{q}$ stabilizer code follows from Corollary [19.

Cyclic codes that contain their euclidean duals can also be nicely characterized in terms of their generator polynomials and defining sets. The following Lemma is a very straight forward extension of the binary case and summarizes some of the known results in the nonbinary case as well, but we include it because of its usefulness in constructing cyclic quantum codes.

Lemma 36. Let $C$ be an $[n, k, d]_{q}$ cyclic code such that $\operatorname{gcd}(n, q)=1$. Let its defining set $Z$ and generator polynomial $g(x)$ be such that any of the following equivalent conditions are satisfied

(i) $x^{n}-1 \equiv 0 \bmod g(x) g^{\dagger}(x)$, where $g^{\dagger}(x)=x^{n-k} g(1 / x)$;

(ii) $Z \subseteq\{-z \mid z \in N \backslash Z\}$;

(iii) $Z \cap Z^{-1}=\emptyset$ where $Z^{-1}=\{-z \bmod n \mid z \in Z\}$.

Then $C^{\perp} \subseteq C$ and there exists an $[[n, 2 k-n, \geq d]]_{q}$ stabilizer code that is pure to $d$.

Proof. The check polynomial of $C$ is given by $h(x)=\left(x^{n}-1\right) / g(x)$, from which we obtain the (un-normalized) generator polynomial of $C^{\perp}$ as $h^{\dagger}(x)=$ $x^{k} h\left(x^{-1}\right)=\left(1-x^{n}\right) /\left(x^{n-k} g\left(x^{-1}\right)\right)=-\left(x^{n}-1\right) / g^{\dagger}(x)$. If $C^{\perp} \subseteq C$, then $g(x) \mid h^{\dagger}(x)$; this means that $g(x)$ divides $\left(x^{n}-1\right) / g^{\dagger}(x)$. In other words $x^{n}-1 \equiv 0 \bmod g(x) g^{\dagger}(x)$.

The defining set of $C^{\perp}$ is given by $\{-z \bmod n \mid z \in N \backslash Z\}$, where $N=\{0,1, \ldots, n-1\}$. Thus, $C^{\perp} \subseteq C$ implies $Z \subseteq\{-z \bmod n \mid N \backslash Z\}$. Since this means that the inverses of elements in $Z$ are present in $N \backslash Z$, this condition can also be written as $Z \cap Z^{-1}=\emptyset$. The existence of quantum code $[[n, 2 k-n, \geq d]]_{q}$ follows from Corollary 21]

Although we have considered purely cyclic codes, a larger class of cyclic quantum codes can be derived by considering constacyclic or conjucyclic codes as in [19], [99].

\section{Cyclic Hamming Codes}

Binary quantum Hamming codes have been studied by various authors; see for instance $[19,35,38]$. We will now derive stabilizer codes from nonbinary classical cyclic Hamming codes. Let $m>1$ be an integer such that $\operatorname{gcd}(q-$ 
$1, m)=1$. A classical cyclic Hamming code $H_{q}(m)$ has parameters $[n, n-$ $m, 3]_{q}$ with length $n=\left(q^{m}-1\right) /(q-1)$. Let $\beta$ denote a primitive $n$th root of unity in $\mathbf{F}_{q^{m}}$. The generator polynomial of $H_{q}(m)$ is given by

$$
g(x)=\prod_{i=0}^{m-1}\left(x-\beta^{q^{i}}\right),
$$

an element of $\mathbf{F}_{q}[x]$. Thus, the code $H_{q}(m)$ is defined by the cyclotomic coset $C_{1}=\left\{q^{i} \bmod n \mid i \in \mathbf{Z}\right\}$.

Lemma 37. The Hamming code $H_{q^{2}}(m)$ contains its hermitian dual, that is, $H_{q^{2}}(m)^{\perp_{h}} \leq H_{q^{2}}(m)$.

Proof. The statement $H_{q^{2}}(m)^{\perp_{h}} \leq H_{q^{2}}(m)$ is equivalent to the fact that the cyclotomic coset $C_{1}$ satisfies $C_{1} \subseteq N_{1}=\left\{-q z \bmod n \mid z \in N \backslash C_{1}\right\}$, where $N=\{0, \ldots, n-1\}$ and $n=\left(q^{2 m}-1\right) /\left(q^{2}-1\right)$. We note that $C_{1}$ can be expressed in the form

$$
C_{1}=\left\{(1-n) q^{2 k} \bmod n \mid k \in \mathbf{Z}\right\}=\left\{-q z q^{2 k} \bmod n \mid k \in \mathbf{Z}\right\},
$$

where $z=q\left(q^{2 m-2}-1\right) /\left(q^{2}-1\right)$. Therefore, the condition $C_{1} \subseteq N_{1}$ holds if and only if $C_{z} \subseteq N \backslash C_{1}$ holds, where $C_{z}=\left\{z q^{2 j} \bmod n \mid j \in \mathbf{Z}\right\}$.

Seeking a contradiction, we assume that the two cyclotomic cosets $C_{1}$ and $C_{z}$ have an element in common, hence are the same. This means that there must exist a positive integer $k$ such that $q^{2 k}=q\left(q^{2 m-2}-1\right) /\left(q^{2}-1\right)$. This implies that $q^{2 k-1}$ divides $q^{2 m-2}-1$, which is absurd. Thus, the sets $C_{1}$ and $C_{z}$ are disjoint, hence $C_{z} \subseteq N \backslash C_{1}$, which proves the claim.

Theorem 38. For each integer $m \geq 2$ such that $\operatorname{gcd}\left(m, q^{2}-1\right)=1$, there exists a pure $[[n, n-2 m, 3]]_{q}$ stabilizer code of length $n=\left(q^{2 m}-1\right) /\left(q^{2}-1\right)$.

Proof. If $\operatorname{gcd}\left(m, q^{2}-1\right)=1$, then there exists a classical $[n, n-m, 3]_{q^{2}}$ Hamming code $H_{q^{2}}(m)$. By Lemma 37] we have $H_{q^{2}}(m)^{\perp_{h}} \leq H_{q^{2}}(m)$, hence there exists a pure $[[n, n-2 m, 3]]_{q}$ stabilizer code by Corollary [19] The purity is due to the fact that the $H_{q^{2}}(m)^{\perp_{h}}$ has minimum distance $q^{2 m-2} \geq 3$ for $m \geq 2$ [52, Theorem 1.8.3].

These quantum Hamming codes are optimal since they attain the quantum Hamming bound, see Corollary 29. A different approach that allows construction of noncyclic perfect quantum codes can be found in [16]. It is also possible to construct quantum codes from Hamming codes that contain their euclidean duals, however these codes do not meet the quantum Hamming bound. 
Lemma 39. If $\operatorname{gcd}(m, q-1)=1$ and $m \geq 2$, then there exists a pure $[[n, n-2 m, 3]]_{q}$ quantum code, where $n=\left(q^{m}-1\right) /(q-1)$.

Proof. The generating polynomial of $[n, n-m, 3]_{q}$ Hamming code, with $\mathrm{n}=\left(q^{m}-1\right) /(q-1)$ is given by equation (11) where $\beta$ is an element of order $n$. The code exists only if $\operatorname{gcd}(m, q-1)=1$. By Lemma 36 a cyclic code contains its dual if $x^{n}-1 \equiv 0 \bmod g(x) g^{\dagger}(x)$, where $g^{\dagger}(x)=x^{n-k} g\left(x^{-1}\right)$. If $g(x)$ is not self-reciprocal then $g(x) g^{\dagger}(x)$ divides $x^{n}-1$ [98]. Since the generating polynomial of the Hamming code is not self-reciprocal, the code contains its euclidean dual. By Lemma 36 we can construct a quantum code with the parameters $[[n, n-2 m, 3]]_{q}$. Once again the purity follows due to the fact the duals of Hamming codes are simplex codes with weight $q^{m-1} \geq 3$ [52, Theorem 1.8.3] for $m \geq 2$.

\section{Quadratic Residue Codes}

Another well known family of classical codes are the quadratic residue codes. Rains constructed quadratic residue codes for prime alphabet in [79]. In this section we will construct two series of quantum codes based on the classical quadratic codes for any arbitrary field using elementary methods.

Theorem 40 (Quadratic Residue Codes). Let $n$ be a prime of the form $n \equiv 3 \bmod 4$, and let $q$ be a power of a prime that is not divisible by $n$. If $q$ is a quadratic residue modulo $n$, then there exists a pure $[[n, 1, d]]_{q}$ stabilizer code with minimum distance $d$ satisfying $d^{2}-d+1 \geq n$.

Proof. Let $\alpha$ denote a primitive $n$th root of unity from some extension field of $\mathbf{F}_{q}$. Let $R=\left\{r^{2} \bmod n \mid r \in \mathbf{Z}\right.$ such that $\left.1 \leq r \leq(n-1) / 2\right\}$ denote the set of quadratic residues modulo $n$. We define the quadratic residue code $C_{R}$ as the cyclic code of length $n$ over $\mathbf{F}_{q}$ that is generated by the polynomial

$$
q(x)=\prod_{r \in R}\left(x-\alpha^{r}\right) .
$$

The code $C_{R}$ has parameters $[n,(n+1) / 2, d]_{q}$ and if $n \equiv 3 \bmod 4$, the dual code $C_{R}^{\perp}$ of $C_{R}$ is given by the cyclic code generated by $(x-1) q(x)$, the evenlike subcode of $C_{R}$. The minimum distance $d$ is bounded by $d^{2}-d+1 \geq n$, see, for instance, [15, pp. 114-119]. Further $\operatorname{wt}\left(C_{R} \backslash C_{R}^{\perp}\right)=\operatorname{wt}\left(C_{R}\right)=d$ by [52, Theorem 6.6.22]. We can deduce from Corollary [21] that there exists a pure $[[n,(n+1)-n, d]]_{q}$ stabilizer code. 
For example, the prime $p=3$ is a quadratic residue modulo $n=23$. The previous proposition guarantees the existence of a $[[23,1, d]]_{3}$ stabilizer code with minimum distance $d \geq 6$.

If $n$ is an odd prime of the form $n \equiv 1 \bmod 4$, then we can also construct quadratic residue codes, but now we need to employ Lemma 20, because $C_{R}$ does not contain its dual.

Theorem 41. Let $n$ be a prime of the form $n \equiv 1 \bmod 4$. Let $q$ be a power of a prime that is not divisible by $n$. If $q$ is a quadratic residue modulo $n$, then there exists a pure $[[n, 1, d]]_{q}$ stabilizer code with minimum distance $d$ bounded from below by $d \geq \sqrt{n}$.

Proof. Let $\alpha$ denote a primitive $n$th root of unity from some extension field of $\mathbf{F}_{q}$. We denote by $R$ denote the set of quadratic residues modulo $n$ and by $N$ the set of quadratic non-residues modulo $n$.

Let $C_{R}$ and $C_{N}$ denote the cyclic codes of length $n$ that are respectively generated by the polynomials $q_{R}(x)$ and $q_{N}(x)$, where

$$
q_{R}(x)=\prod_{r \in R}\left(x-\alpha^{r}\right) \quad \text { and } \quad q_{N}(x)=\prod_{r \in N}\left(x-\alpha^{r}\right) .
$$

Both codes have parameters $[n,(n+1) / 2, d]_{q}$ with $d^{2} \geq n$, see $[15$, pp. 114119].

The dual code of $C_{R}$ is given by the even-like subcode of $C_{N}$; in other words, $C_{R}^{\perp}$ is a cyclic code of length $n$ over $\mathbf{F}_{q}$ that is generated by the polynomial $(x-1) q_{N}(x)$; in particular, $C_{R}^{\perp} \leq C_{N}$. Moreover $\operatorname{wt}\left(C_{R} \backslash C_{N}^{\perp}\right)=$ $\operatorname{wt}\left(C_{N} \backslash C_{R}^{\perp}\right)=d$ by [52, Theorem 6.6.22]. Therefore, we obtain a pure $[[n,(n+1) / 2+(n+1) / 2-n, d]]_{q}$ code by Lemma 20.

\section{Quantum Melas Codes}

One of the earliest family of codes that were constructed with a view to correcting burst errors are the Melas codes. While not as well known as the Hamming codes or the quadratic residue codes, they are nonetheless an interesting family of codes. These codes have been well investigated, especially in the mathematical community, because of their connections to algebraic geometry $[62,87,88,97]$. See [51] for an interesting read on the connections to number theory.

The Melas code ${ }^{*} \mathcal{M}_{q}(m)$ is a cyclic $[n, n-2 m, \geq 3]_{q}$ code with $n=q^{m}-1$.

${ }^{*}$ The classical Melas codes are defined over a prime field $\mathbf{F}_{p}$ and have the parameters $\left[p^{m}-1, p^{m}-m-1, \geq 3\right]_{p}$ (cf. [95]); here we consider a generalization to arbitrary finite fields. 
The generator polynomial of $\mathcal{M}_{q}(m)$ is given by

$$
g(x)=\prod_{i=0}^{m-1}\left(x-\alpha^{q^{i}}\right)\left(x-\alpha^{-q^{i}}\right),
$$

where $\alpha$ is a primitive element in $\mathbf{F}_{q^{m}}$. Alternatively, the defining set of the code is given by $Z=C_{1} \cup C_{-1}=\left\{ \pm q^{i} \bmod n \mid 0 \leq i<m\right\}$.

Lemma 42. The Melas code $\mathcal{M}_{q^{2}}(m)$ contains its hermitian dual.

Proof. By Lemma [35] it suffices to show that $Z \cap Z^{-q}=\emptyset$. Seeking a contradiction, we assume that $Z \cap Z^{-q} \neq \emptyset$. Since $\operatorname{gcd}\left(q^{2}, q^{2 m}-1\right)=1$, this implies that there must exist some integer $i$ in the range $0 \leq i<m$ such that $q^{2 i} \equiv \pm q \bmod n$, but that is impossible; so $Z \cap Z^{-q}=\emptyset$.

Lemma 43. If $q$ is even, then the minimum distance of the Melas code $\mathcal{M}_{q^{2}}(m)$ is at least 3 .

Proof. The parity check matrix of $\mathcal{M}_{q^{2}}(m)$ is given by

$$
H=\left(\begin{array}{ccccc}
1 & \alpha & \alpha^{2} & \cdots & \alpha^{n-1} \\
1 & \alpha^{-1} & \alpha^{-2} & \cdots & \alpha^{-(n-1)}
\end{array}\right)
$$

This matrix has a rank 2 only if no two columns are scalar multiples of each other. Seeking a contradiction, we suppose that

$$
\left(\begin{array}{c}
\alpha^{x} \\
\alpha^{-x}
\end{array}\right)=\alpha^{t}\left(\begin{array}{c}
\alpha^{y} \\
\alpha^{-y}
\end{array}\right)
$$

holds for distinct $x$ and $y$. This yields $\alpha^{2 t}=1$, which implies $t \in\{0, n / 2\}$. If $q$ is even, then $n$ is odd, and so $t$ cannot equal $n / 2$. If $t=0$, then $x=y$ contradicting the distinctness of $x$ and $y$. Therefore, we can conclude that $H$ has rank $r=2$; thus, the minimum distance is at least 3 .

Theorem 44 (Quantum Melas codes). If $q$ is even and $n=q^{2 m}-1$, there exist quantum Melas codes with parameters $[[n, n-4 m, \geq 3]]_{q}$ that is pure to 3.

Proof. By Lemma 42 we have $\mathcal{M}_{q^{2}}(m)^{\perp_{h}} \subseteq \mathcal{M}_{q^{2}}(m)$ and by Lemma 43 we have the distance $\geq 3$. So by Corollary 19 there exists an $[[n, n-4 m, \geq 3]]_{q}$ quantum code. 


\section{Quantum BCH Codes}

In this section we consider a popular family of classical codes, the $\mathrm{BCH}$ codes, and construct the associated nonbinary quantum stabilizer codes. Binary quantum BCH codes were studied in $[19,29,45,94]$. The CSS construction turns out to be especially useful, because $\mathrm{BCH}$ codes form a naturally nested family of codes. In case of primitive $\mathrm{BCH}$ codes over prime fields the distance of the dual is lower bounded by the generalized Carlitz-Uchiyama bound, and this allows us to derive bounds on the minimum distance of the resulting quantum codes.

Let $q$ be a power of a prime and $n$ a positive integer that is coprime to $q$. Recall that a BCH code $C$ of length $n$ and designed distance $\delta$ over $\mathbf{F}_{q}$ is a cyclic code whose defining set $Z$ is given by a union of $\delta-1$ subsequent cyclotomic cosets,

$$
Z=\bigcup_{x=b}^{b+\delta-2} C_{x}, \quad \text { where } \quad C_{x}=\left\{x q^{r} \bmod n \mid r \in \mathbf{Z}, r \geq 0\right\}
$$

The generator polynomial of the code is of the form

$$
g(x)=\prod_{z \in Z}\left(x-\beta^{z}\right),
$$

where $\beta$ is a primitive $n$-th root of unity of some extension field of $\mathbf{F}_{q}$. The definition ensures that $g(x)$ generates a cyclic $[n, k, d]_{q}$ code of dimension $k=n-|Z|$ and minimum distance $d \geq \delta$. If $b=1$, then the code $C$ is called a narrow-sense $\mathrm{BCH}$ code, and if $n=q^{m}-1$ for some $m \geq 1$, then the code is called primitive.

Generalized Carlitz-Uchiyama Bound. Our first construction derives stabilizer codes from BCH codes over prime fields. We use the Knuth-Iverson bracket [statement] in the formulation of the Carlitz-Uchiyama bound that evaluates to 1 if statement is true and 0 otherwise.

Lemma 45 (Generalized Carlitz-Uchiyama Bound). Let $p$ be a prime. Let $C$ denote a narrow-sense $B C H$ code of length $n=p^{m}-1$ over $\mathbf{F}_{p}$, of designed distance $\delta=2 t+1$. Then the minimum distance $d^{\perp}$ of its euclidean dual code $C^{\perp}$ is bounded by

$$
d^{\perp} \geq\left(1-\frac{1}{p}\right)\left(p^{m}-\frac{\delta-2-[\delta-1 \equiv 0 \bmod p]}{2}\left\lfloor 2 p^{m / 2}\right\rfloor\right) .
$$


Proof. See [95, Theorem 7]; for further background, see [67, page 280].

Theorem 46. Let $p$ be a prime. Let $C$ be a $\left[p^{m}-1, k, \geq \delta\right]_{p}$ narrow-sense $B C H$ code of designed distance $\delta=2 t+1$ and $C^{*} a\left[p^{m}-1, k^{*}, d^{*}\right]_{p} B C H$ code such that $C \subseteq C^{*}$. Then there exists a $\left[\left[p^{m}-1, k^{*}-k, \geq \min \left\{d^{*}, d^{\perp}\right\}\right]\right]_{p}$ stabilizer code, where $d^{\perp}$ is given by (16).

Proof. The result follows from applying Lemma 45 to $C$ and Lemma 20 to the codes $C$ and $C^{*}$.

Remark 47. (i) The Carlitz-Uchiyama bound becomes trivial for larger design distances. (ii) In [72, Corollary 2] it was shown that for binary $\mathrm{BCH}$ codes of design distance $d$, the lower bound in equation (16) is attained when $n=2^{2 a b}-1$, where $a$ is the smallest integer such that $d-2 \mid 2^{a}+1$ and $b$ is odd. (iii) For a further tightening of the Carlitz-Uchiyama bound see [73, Theorem 2].

Primitive $\mathrm{BCH}$ codes containing their duals. We can extend result of the previous section to $\mathrm{BCH}$ codes over finite fields that are not necessarily prime. In fact, if we restrict ourselves to smaller designed distances, then we can even achieve significantly sharper results. We will just review the results and refer the reader to our companion paper [2] for the proofs.

In the $\mathrm{BCH}$ code construction, it is in general not obvious how large the cyclotomic cosets will be. However, if the designed distance is small, then one can show that the cyclotomic cosets all have maximal size.

Lemma 48. A narrow-sense, primitive $B C H$ code with design distance $2 \leq$ $\delta \leq q^{\lceil m / 2\rceil}+1$ has parameters $\left[q^{m}-1, q^{m}-1-m\lceil(\delta-1)(1-1 / q)\rceil, \geq \delta\right]_{q}$.

Proof. See [2, Theorem A]; the binary case was already established by Steane [94].

In the case of small designed distances, primitive, narrow-sense $\mathrm{BCH}$ codes contain their euclidean duals.

Lemma 49. A narrow-sense, primitive BCH code over $\mathbf{F}_{q}^{n}$ contains its euclidean dual if and only if its design distance $2 \leq \delta \leq q^{\lceil m / 2\rceil}-1-(q-$ $2)\left[m\right.$ odd], where $n=q^{m}-1$ and $m \geq 2$.

Proof. See [2, Theorem C].

A simple consequence is the following theorem: 
Theorem 50. If $C$ is a narrow-sense primitive $B C H$ code over $\mathbf{F}_{q}$ with design distance $2 \leq \delta \leq q^{\lceil m / 2\rceil}-1-(q-2)[m$ odd $]$ and $m \geq 2$, then there exists an $\left[\left[q^{m}-1, q^{m}-1-2 m\lceil(\delta-1)(1-1 / q)\rceil, \geq \delta\right]\right]_{q}$ stabilizer code that is pure to $\delta$.

Proof. If we combine Lemmas 48 and 49 and apply the CSS construction, then we obtain the claim.

One can argue in a similar way for hermitian duals of primitive, narrowsense BCH codes.

Theorem 51. If $C$ is a narrow-sense primitive $B C H$ code over $\mathbf{F}_{q^{2}}^{n}$ with design distance $2 \leq \delta \leq q^{m}-1$, then there exists an $\left[\left[q^{2 m}-1, q^{2 m}-1-\right.\right.$ $\left.\left.2 m\left\lceil(\delta-1)\left(1-1 / q^{2}\right)\right\rceil, \geq \delta\right]\right]_{q}$ stabilizer code that is pure to $\delta$.

Proof. See [2] for details.

When $m=1$, the $\mathrm{BCH}$ codes are the same as the Reed Solomon codes and this case has been dealt with in [47]. An alternate perspective using Reed-Muller codes is considered in [84].

Extending quantum BCH codes. It is not always possible to extend a stabilizer code, because the corresponding classical codes are required to be self-orthogonal. In this paragraph we will show that it is possible to extend narrow-sense $\mathrm{BCH}$ codes of certain lengths.

Lemma 52. Let $\mathbf{F}_{q^{2}}$ be a finite field of characteristic $p$. If $C$ is a narrowsense $[n, k, \geq d]_{q^{2}} B C H$ code such that $C^{\perp_{h}} \subseteq C$ and $n \equiv-1 \bmod p$, then there exists an $[[n, 2 k-n, \geq d]]_{q}$ stabilizer code that is pure to $d$ which can be extended to an $[[n+1,2 k-n-1, \geq d+1]]_{q}$ stabilizer code that is pure to $d+1$.

Proof. Since $C^{\perp_{h}} \subseteq C$, Corollary 19$]$ implies the existence of an [[n, $2 k-n, \geq$ $d]]_{q}$ quantum code that is pure to $d$. And being narrow-sense the parity check matrix of $C$ has the form

$$
H=\left(\begin{array}{ccccc}
1 & \alpha & \alpha^{2} & \cdots & \alpha^{(n-1)} \\
1 & \alpha^{2} & \alpha^{2(2)} & \cdots & \alpha^{2(n-1)} \\
\vdots & \ddots & \ddots & \ddots & \ddots \\
1 & \alpha^{d-1} & \alpha^{2(d-1)} & \cdots & \alpha^{(n-1)(d-1)}
\end{array}\right)
$$


where $\alpha$ is a primitive $n^{\text {th }}$ root of unity. This can be extended to give an $[n+1, k, d+1]$ code $C_{e}$, whose parity check matrix is given as

$$
H_{e}=\left(\begin{array}{cccccc}
1 & 1 & 1 & \cdots & 1 & 1 \\
1 & \alpha & \alpha^{2} & \cdots & \alpha^{(n-1)} & 0 \\
1 & \alpha^{2} & \alpha^{2(2)} & \cdots & \alpha^{2(n-1)} & 0 \\
\vdots & \ddots & \ddots & \ddots & \ddots & \vdots \\
1 & \alpha^{d-1} & \alpha^{2(d-1)} & \cdots & \alpha^{(n-1)(d-1)} & 0
\end{array}\right) .
$$

We will show that $C_{e}^{\perp_{h}}$ is self-orthogonal. Let $R_{i}$ be the $i^{\text {th }}$ row in $H_{e}$. For $2 \leq i \leq d$ the self-orthogonality of $H$ implies that $\left\langle R_{i} \mid R_{j}\right\rangle_{h}=0$. We need to show that $\left\langle R_{i} \mid \mathbf{1}\right\rangle_{h}=0,1 \leq i \leq d$. For $2 \leq i \leq d$ we have $\left\langle R_{i} \mid \mathbf{1}\right\rangle_{h}=$ $\sum_{j=0}^{n-1} \alpha^{i j}=\left(\alpha^{i n}-1\right) /\left(\alpha^{i}-1\right)=0$, as $\alpha^{n}=1$ and $\alpha^{i} \neq 1$. For $i=1$ we have $\langle\mathbf{1} \mid \mathbf{1}\rangle_{h}=n+1 \bmod p$, which vanishes because of the assumption $n \equiv-1$ $\bmod p$.

Now we will show that the rank of $H_{e}$ is $d$, thus $C_{e}$ has a minimum distance of at least $d+1$. Any $d$ columns of $H_{e}$ excluding the last column form a $d \times d$ vandermonde matrix which is nonsingular, indicating that the $d$ columns are linearly independent. If we consider any set of $d$ columns that includes the last column, we can find the determinant of the corresponding matrix by expanding by the last column. This gives us a $d-1 \times d-1$ vandermonde matrix with nonzero determinant. Thus any $d$ columns of $H_{e}$ are independent and the minimum distance of $C_{e}$ is at least $d+1$. Therefore $C_{e}$ is an $[n+1, k, \geq d+1]_{q^{2}}$ extended cyclic code such that $C_{e}^{\perp_{h}} \subseteq C_{e}$. By Corollary [19] it defines an $[[n+1,2 k-n-1, \geq d+1]]_{q}$ quantum code pure to $d+1$.

Corollary 53. For all prime powers $q$, integers $m \geq 1$ and all $\delta$ in the range $2 \leq \delta \leq q^{m}-1$ there exists an

$$
\left[\left[q^{2 m}, q^{2 m}-2-2 m\left\lceil(\delta-1)\left(1-1 / q^{2}\right)\right\rceil, \geq \delta+1\right]\right]_{q}
$$

stabilizer code pure to $\delta+1$.

Proof. The stabilizer codes from Theorem 51 are derived from primitive, narrow-sense BCH codes. If $p$ denotes the characteristic of $\mathbf{F}_{q^{2}}$, then $q^{2 m}$ $1 \equiv-1 \bmod p$, so the stabilizer codes given in Theorem [51 can be extended by Lemma 52 .

A result similar to Lemma 52 can be developed for $\mathrm{BCH}$ codes that contain their euclidean duals. 


\section{Puncturing Stabilizer Codes}

If we delete one coordinate in all codewords of a classical code, then we obtain a shorter code that is called the punctured code. In general, we cannot proceed in the same way with stabilizer codes, since the resulting matrices might not commute if we delete one or more tensor components.

Rains [79] invented an interesting approach that solves the puncturing problem for linear stabilizer codes and, even better, gives a way to construct stabilizer codes from arbitrary linear codes. The idea is to associate with a classical linear code a so-called puncture code; if the puncture code contains a codeword of weight $r$, then a self-orthogonal code of length $r$ exists and the minimum distance is the same or higher than that of the initial classical code. Further convenient criteria for puncture codes were given in [47].

In this section, we generalize puncturing to arbitrary stabilizer codes and review some known facts. Determining a puncture code is a challenging task, and we conclude this section by showing how to puncture quantum $\mathrm{BCH}$ codes.

The Puncture Code. It will be convenient to denote the the pointwise product of two vectors $u$ and $v$ in $\mathbf{F}_{q}^{n}$ by $u v$, that is, $u v=\left(u_{i} v_{i}\right)_{i=1}^{n}$.

Suppose that $C \leq \mathbf{F}_{q}^{2 n}$ is an arbitrary additive code. The associated puncture code $\mathrm{P}_{s}(C) \subseteq \mathbf{F}_{q}^{n}$ is defined as

$$
\mathrm{P}_{s}(C)=\left\{\left(b_{k} a_{k}^{\prime}-b_{k}^{\prime} a_{k}\right)_{k=1}^{n} \mid(a \mid b),\left(a^{\prime} \mid b^{\prime}\right) \in C\right\}^{\perp} .
$$

Theorem 54. Suppose that $C$ is an arbitrary additive subcode of $\mathbf{F}_{q}^{2 n}$ of size $|C|=q^{n} / K$ such that $\operatorname{swt}\left(C^{\perp_{s}} \backslash C\right)=d$. If the puncture code $\mathrm{P}_{s}(C)$ contains a codeword of Hamming weight $r$, then there exists an $\left(\left(r, K^{*}, d^{*}\right)\right)_{q}$ stabilizer code with $K^{*} \geq K / q^{n-r}$ that has minimum distance $d^{*} \geq d$ when $K^{*}>1$. If $\operatorname{swt}\left(C^{\perp_{s}}\right)=d$, then the resulting punctured stabilizer code is pure to $d$.

Proof. Let $x$ be a codeword of weight $r$ in the $\mathrm{P}_{s}(C)$. Define an additive code $C_{x} \leq \mathbf{F}_{q}^{2 n}$ by

$$
C_{x}=\{(a \mid b x) \mid(a \mid b) \in C\} .
$$

If $(a \mid b x)$ and $\left(a^{\prime} \mid b^{\prime} x\right)$ are arbitrary elements of $C_{x}$, then

$$
\left\langle(a \mid b x) \mid\left(a^{\prime} \mid b^{\prime} x\right)\right\rangle_{s}=\operatorname{tr}\left(\sum_{k=1}^{n}\left(b_{k} a_{k}^{\prime}-b_{k}^{\prime} a_{k}\right) x_{k}\right)=0
$$


by definition of $\mathrm{P}_{s}(C)$; thus, $C_{x} \leq\left(C_{x}\right)^{\perp_{s}}$.

Let $C_{x}^{R}=\left\{\left(a_{k} \mid b_{k}\right)_{k \in S} \mid(a \mid b) \in C_{x}\right\}$ denote the restriction of $C_{x}$ to the support $S$ of the vector $x$. Since equation (19) depends only on the nonzero coefficients of the vector $x$, it follows that $C_{x}^{R} \leq\left(C_{x}^{R}\right)^{\perp_{s}}$ holds.

We note that $|C| \geq\left|C_{x}^{R}\right|$; hence, the dimension $K^{*}$ of the punctured quantum code is bounded by

$$
K^{*} \geq q^{r} /\left|C_{x}^{R}\right| \geq q^{r} /|C|=q^{r} /\left(q^{n} / K\right)=K / q^{n-r} .
$$

It remains to show that $\operatorname{swt}\left(\left(C_{x}^{R}\right)^{\perp_{s}} \backslash C_{x}^{R}\right) \geq d$. Seeking a contradiction, we suppose that $u_{x}^{R}$ is a vector in $\left(C_{x}^{R}\right)^{\perp_{s}} \backslash C_{x}^{R}$ such that $\operatorname{swt}\left(u_{x}^{R}\right)<d$. Let $u_{x}=(a \mid b)$ denote the vector in $\left(C_{x}\right)^{\perp_{s}}$ that is zero outside the support of $x$ and coincides with $u_{x}^{R}$ when restricted to the support of $x$. It follows that $(a x \mid b)$ is contained in $C^{\perp_{s}}$. However $\operatorname{swt}(a x \mid b)<d$, so $(a x \mid b)$ must be an element of $C$, since $\operatorname{swt}\left(C^{\perp_{s}} \backslash C\right)=d$. This implies that $(a x \mid b x)$ is an element of $C_{x} \leq\left(C_{x}\right)^{\perp_{s}}$. Arguing as before, it follows that $\left(a x^{2} \mid b x\right)$ is in $C$ and $\left(a x^{2} \mid b x^{2}\right)$ is in $C_{x}$. Repeating the process, we obtain that $v_{x}=\left(a x^{q-1} \mid b x^{q-1}\right)$ is in $C_{x}$, and we note that $x^{q-1}$ is the characteristic vector of the support of $x$. Restricting $v_{x}$ in $C_{x}$ to the support of $x$ yields $u_{x}^{R} \in C_{x}^{R}$, contradicting the assumption that $u_{x}^{R} \in\left(C_{x}^{R}\right)^{\perp_{s}} \backslash C_{x}^{R}$.

Finally, the last statement concerning the purity is easy to prove (a direct generalization of the argument given in [47] for pure linear codes).

If the code $C$ is a direct product, as in the case of CSS codes, then the expression for the puncture code simplifies somewhat.

Lemma 55. If $C_{1}$ and $C_{2}$ are two additive subcodes of $\mathbf{F}_{q}^{n}$, then

$$
\mathrm{P}_{s}\left(C_{1} \times C_{2}\right)=\left\{a b \mid a \in C_{1}, b \in C_{2}\right\}^{\perp} \leq \mathbf{F}_{q}^{n} .
$$

Proof. Since $\left\langle a b \mid a \in C_{1}, b \in C_{2}\right\rangle=\left\langle\left(b a^{\prime}-b^{\prime} a\right) \mid a, a^{\prime} \in C_{1}, b, b^{\prime} \in C_{2}\right\rangle$, the claim about the orthogonal complements of these sets is obvious.

Since many quantum codes are constructed from self-orthogonal codes $C \leq C^{\perp}$, we write shortly

$$
\mathrm{P}_{e}(C)=\mathrm{P}_{s}(C \times C)=\{a b \mid a, b \in C\}^{\perp} .
$$

Puncturing $\mathbf{B C H}$ Codes. In this section, we let $\mathrm{BCH}_{q}^{m}(\delta)$ denote a primitive, narrow-sense $q$-ary BCH code of length $n=q^{m}-1$ and designed distance $\delta$. We will illustrate the previous result by puncturing such $\mathrm{BCH}$ codes. Some knowledge about the puncture code is necessary for this task, 
and we show in Theorem 57 that a cyclic generalized Reed-Muller code is contained in the puncture code.

First, let us recall some basic facts about cyclic generalized Reed-Muller codes, see $[9,10,53,75]$ for details. Let $L_{m}(\nu)$ denote the subspace of $\mathbf{F}_{q}\left[x_{1}, \ldots, x_{m}\right]$ of polynomials of degree $\leq \nu$, and let $\left(P_{0}, \ldots, P_{n-1}\right)$ be an enumeration of the points in $\mathbf{F}_{q}^{m}$ where $P_{0}=\mathbf{0}$. The $q$-ary cyclic generalized Reed-Muller code $\mathcal{R}_{q}^{*}(\nu, m)$ of order $\nu$ and length $n=q^{m}-1$ is defined as

$$
\mathcal{R}_{q}^{*}(\nu, m)=\left\{e v f \mid f \in L_{m}(\nu)\right\},
$$

where the codewords are evaluations of the polynomials in all but $P_{0}$, ev $f=$ $\left(f\left(P_{1}\right), \ldots, f\left(P_{n-1}\right)\right)$. The dimension $k^{*}(\nu)$ of the code $\mathcal{R}_{q}^{*}(\nu, m)$ is given by the formula $k^{*}(\nu)=\sum_{j=0}^{m}(-1)^{j}\left(\begin{array}{c}m \\ j\end{array}\right)\left(\begin{array}{c}m+\nu-j q \\ \nu-j q\end{array}\right)$, and its minimum distance $d^{*}(\nu)=(R+1) q^{Q}-1$, where $m(q-1)-\nu=(q-1) Q+R$ with $0 \leq R<q-1$. The dual code of $\mathcal{R}_{q}^{*}(\nu, m)$ can be characterized by

$$
\mathcal{R}_{q}^{*}(\nu, m)^{\perp}=\left\{e v f \mid f \in L_{m}^{*}\left(\nu^{\perp}\right)\right\},
$$

where $\nu^{\perp}=m(q-1)-\nu-1$ and $L_{m}^{*}(\nu)$ is the subspace of all nonconstant polynomials in $L_{m}(\nu)$;

It is well-known that a primitive, narrow-sense $\mathrm{BCH}$ code contains a cyclic generalized Reed-Muller code, see [53, Theorem 5], and we determine the largest such subcode in our next lemma.

Lemma 56. We have $\mathcal{R}_{q}^{*}(\nu, m) \subseteq \mathrm{BCH}_{q}^{m}(\delta)$ for $\nu=(m-Q)(q-1)-R$, with $Q=\left\lfloor\log _{q}(\delta+1)\right\rfloor$ and $R=\left\lceil(\delta+1) / q^{Q}\right\rceil-1$. For all orders $\nu^{\prime}>\nu$, we have $\mathcal{R}_{q}^{*}\left(\nu^{\prime}, m\right) \nsubseteq \mathrm{BCH}_{q}^{m}(\delta)$.

Proof. First, we show that $\mathcal{R}_{q}^{*}(\nu, m) \subseteq \mathrm{BCH}_{q}^{m}(\delta)$. Recall that the minimum distance $d^{*}(\nu)=(R+1) q^{Q}-1$, where $m(q-1)-\nu=(q-1) Q+R$ with $0 \leq$ $R<q-1$. By [53, Theorem 5], we have $\mathcal{R}_{q}^{*}(\nu, m) \subseteq \mathrm{BCH}_{q}^{m}\left((R+1) q^{Q}-1\right)$. Notice that $(R+1) q^{Q}-1=\left\lceil(\delta+1) / q^{Q}\right\rceil q^{Q}-1 \geq \delta$, so $\mathrm{BCH}_{q}^{m}\left((R+1) q^{Q}-1\right) \subseteq$ $\mathrm{BCH}_{q}^{m}(\delta)$. Therefore, $\mathcal{R}_{q}^{*}(\nu, m) \subseteq \mathrm{BCH}_{q}^{m}(\delta)$, as claimed.

For the second claim, it suffices to show that $\mathcal{R}_{q}^{*}(\nu+1, m)$ is not a subcode of $\mathrm{BCH}_{q}^{m}(\delta)$. We will prove this by showing that the minimum distance $d^{*}(\nu+1)<\delta$. Notice that

$$
m(q-1)-(\nu+1)= \begin{cases}(q-1) Q+R-1 & \text { for } R \geq 1, \\ (q-1)(Q-1)+q-2 & \text { for } R=0 .\end{cases}
$$

with $R$ and $Q$ as given in the hypothesis. Therefore, the distance $d^{*}(\nu+1)$ of $\mathcal{R}_{q}^{*}(\nu+1, m)$ is given by

$$
d^{*}(\nu+1)= \begin{cases}\left(\left\lceil(\delta+1) / q^{Q}\right\rceil-1\right) q^{Q}-1 & \text { for } R \geq 1 \\ (q-1) q^{Q-1}-1 & \text { for } R=0 .\end{cases}
$$


In both cases, it is straightforward to verify that $d^{*}(\nu+1)<\delta$.

Explicitly determining the puncture code is a challenging task. For the duals of $\mathrm{BCH}$ codes, we are able to determine large subcodes of the puncture code.

Theorem 57. If $\delta<q^{\lfloor m / 2\rfloor}-1$, then $\mathcal{R}_{q}^{*}(\mu, m) \subseteq \mathrm{P}_{e}\left(\mathrm{BCH}_{q}^{m}(\delta)^{\perp}\right)$ for all orders $\mu$ in the range $0 \leq \mu \leq m(q-1)-2(R+(q-1) Q)+1$ with $Q=$ $\left\lfloor\log _{q}(\delta+1)\right\rfloor$ and $R=\left\lceil(\delta+1) / q^{Q}\right\rceil-1$.

Proof. By Lemma [56, we have $\mathcal{R}_{q}^{*}(\nu, m) \subseteq \mathrm{BCH}_{q}^{m}(\delta)$ for $\nu=(m-Q)(q-$ 1) $-R$; hence, $\mathrm{BCH}_{q}^{m}(\delta)^{\perp} \subseteq \mathcal{R}_{q}^{*}(\nu, m)^{\perp}$. It follows from the definition of the puncture code that $\mathrm{P}_{e}\left(\mathrm{BCH}_{q}^{m}(\delta)^{\perp}\right) \supseteq \mathrm{P}_{e}\left(\mathcal{R}_{q}^{*}(\nu, m)^{\perp}\right)$. However,

$$
\begin{aligned}
\mathrm{P}_{e}\left(\mathcal{R}_{q}^{*}(\nu, m)^{\perp}\right) & =\left\{\text { evf } \cdot \text { ev } g \mid f, g \in L_{m}^{*}\left(\nu^{\perp}\right)\right\}^{\perp}, \\
& \supseteq\left\{e v f \mid f \in L_{m}^{*}\left(2 \nu^{\perp}\right)\right\}^{\perp}=\mathcal{R}_{q}^{*}\left(\left(2 \nu^{\perp}\right)^{\perp}, m\right),
\end{aligned}
$$

where the last equality follows from equation (21). This is meaningful only if $\left(2 \nu^{\perp}\right)^{\perp} \geq 0$ or, equivalently, if $\nu \geq(m(q-1)-1) / 2$. Since $\delta<q^{\lfloor m / 2\rfloor}-1$, it follows that $Q \leq\lfloor m / 2\rfloor-1$, and the order $\nu$ satisfies

$$
\begin{aligned}
\nu & =(m-Q)(q-1)-R \geq\lceil m / 2+1\rceil(q-1)-R \\
& \geq\lceil m / 2\rceil(q-1)+1 \geq(m(q-1)-1) / 2,
\end{aligned}
$$

as required. Since $\mathcal{R}_{q}^{*}(\mu, m) \subseteq \mathcal{R}_{q}^{*}\left(\left(2 \nu^{\perp}\right)^{\perp}, m\right)$ for $0 \leq \mu \leq\left(2 \nu^{\perp}\right)^{\perp}$, we have $\mathcal{R}_{q}^{*}(\mu, m) \subseteq \mathrm{P}_{e}\left(\mathrm{BCH}_{q}^{m}(\delta)^{\perp}\right)$.

Unfortunately, the weight distribution of generalized cyclic Reed-Muller codes is not known, see [22]. However, we know that the puncture code of $\mathrm{BCH}_{q}^{m}(\delta)^{\perp}$ contains the codes $\mathcal{R}_{q}^{*}(0, m) \subseteq \mathcal{R}_{q}^{*}(1, m) \subseteq \cdots \subseteq \mathcal{R}_{q}^{*}(m(q-$ $1)-2(R+(q-1) Q)+1, m)$, so it must contain codewords of the respective minimum distances.

Corollary 58. If $\delta$ and $\mu$ are integers in the range $2 \leq \delta<q^{\lfloor m / 2\rfloor}-1$ and $0 \leq \mu \leq m(q-1)-2(R+(q-1) Q)+1$, where $Q=\left\lfloor\log _{q}(\delta+1)\right\rfloor$ and $R=\left\lceil(\delta+1) / q^{Q}\right\rceil-1$, then there exists a

$$
\left[\left[d^{*}(\mu), \geq d^{*}(\mu)-2 m\lceil(\delta-1)(1-1 / q)\rceil, \geq \delta\right]\right]_{q}
$$

stabilizer code of length $d^{*}(\mu)=(\rho+1) q^{\sigma}-1$, where $\sigma$ and $\rho$ satisfy the relations $m(q-1)-\mu=(q-1) \sigma+\rho$ and $0 \leq \rho<q-1$. 
Proof. If $2 \leq \delta<q^{\lfloor m / 2\rfloor}-1$, then from Theorem [50] we know that there exists an $\left[\left[q^{m}-1, q^{m}-1-2 m\lceil(\delta-1)(1-1 / q)\rceil, \geq \delta\right]\right]_{q}$ quantum code. From Lemma 57 we know that $\mathrm{P}_{e}\left(\mathrm{BCH}_{q}^{m}(\delta)^{\perp}\right) \supseteq \mathcal{R}_{q}^{*}(\mu, m)$, where $0 \leq$ $\mu \leq m(q-1)-2(q-1) Q-2 R+1$. By Theorem [54 if there exists a vector of weight $r$ in $\mathrm{P}_{e}\left(\mathrm{BCH}_{q}^{m}(\delta)^{\perp}\right)$, the corresponding quantum code can be punctured to give $[[r, \geq r-2 m\lceil(\delta-1)(1-1 / q)\rceil), d \geq \delta]]_{q}$. The minimum distance of $\mathcal{R}_{q}^{*}(\mu, m)$ is $d^{*}(\mu)=(\rho+1) q^{\sigma}-1$, where $0 \leq \rho<q-1[53$, Theorem 5]. Hence, it is always possible to puncture the quantum code to $\left[\left[d^{*}(\mu), \geq d^{*}(\mu)-2 m\lceil(\delta-1)(1-1 / q)], \geq \delta\right]\right]_{q}$.

It is also possible to puncture quantum codes constructed via classical codes self-orthogonal with respect to the hermitian inner product. Examples of such puncturing can be found in [47] and [84].

\section{MDS Codes}

A quantum code that attains the quantum Singleton bound is called a quantum Maximum Distance Separable code or quantum MDS code for short. These codes have received much attention, but many aspects have not yet been explored in the quantum case (but see $[47,79]$ ). In this section we will study the maximal length of MDS stabilizer codes.

An interesting result concerning the purity of quantum MDS codes was derived by Rains [79, Theorem 2]:

Lemma 59 (Rains). An $[[n, k, d]]_{q}$ quantum $M D S$ code with $k \geq 1$ is pure up to $n-d+2$.

Corollary 60. All quantum MDS codes are pure.

Proof. An $[[n, k, d]]_{q}$ quantum MDS code with $k=0$ is pure by definition; if $k \geq 1$ then it is pure up to $n-d+2$. By the quantum Singleton bound $n-2 d+2=k \geq 0$; thus, $n-d+2 \geq d$, which means that the code is pure.

Lemma 61. For any $[[n, n-2 d+2, d]]_{q}$ quantum MDS stabilizer code with $n-2 d+2>0$ the corresponding classical codes $C \subseteq C^{\perp_{a}}$ are also MDS.

Proof. If an $[[n, n-2 d+2, d]]_{q}$ stabilizer code exists, then Theorem [15] implies the existence of an additive $[n, d-1]_{q^{2}}$ code $C$ such that $C \subseteq C^{\perp_{a}}$. Corollary 60] shows that $C^{\perp_{a}}$ has minimum distance $d$, so $C^{\perp_{a}}$ is an $[n, n-d+$ $1, d]_{q^{2}}$ MDS code. By Lemma [59], the minimum distance of $C$ is $\geq n-d+2$, so $C$ is an $[n, d-1, n-d+2]_{q^{2}}$ MDS code. 
A classical $[n, k, d]_{q}$ MDS code is said to be trivial if $k \leq 1$ or $k \geq n-1$. A trivial MDS code can have arbitrary length, but a nontrivial one cannot. The next lemma is a straightforward generalization from linear to additive MDS codes.

Lemma 62. Assume that there exists a classical additive $\left(n, q^{k}, d\right)_{q} M D S$ code $C$.

(i) If the code is trivial, then it can have arbitrary length.

(ii) If the code is nontrivial, then its code parameters must be in the range $2 \leq k \leq \min \{n-2, q-1\}$ and $n \leq q+k-1 \leq 2 q-2$.

Proof. The first statement is obvious. For (ii), we note that the weight distribution of the code $C$ and its dual are related by the MacWilliams relations. The proof given in [67, p. 320-321] for linear codes applies without change, and one finds that the number of codewords of weight $n-k+2$ in $C$ is given by

$$
A_{n-k+2}=\left(\begin{array}{c}
n \\
k-2
\end{array}\right)(q-1)(q-n+k-1) .
$$

Since $A_{n-k+2}$ must be a nonnegative number, we obtain the claim.

We say that a quantum $[[n, k, d]]_{q}$ MDS code is trivial if and only if its minimum distance $d \leq 2$. The length of trivial quantum MDS codes is not bounded, but the length of nontrivial ones is, as the next lemma shows.

Theorem 63 (Maximal Length of MDS Stabilizer Codes). A nontrivial $[[n, k, d]]_{q}$ MDS stabilizer code satisfies the following constraints:

i) its length $n$ is in the range $4 \leq n \leq q^{2}+d-2 \leq 2 q^{2}-2$;

ii) its minimum distance satisfies $\max \left\{3, n-q^{2}+2\right\} \leq d \leq \min \left\{n-1, q^{2}\right\}$.

Proof. By definition, a quantum MDS code attains the Singleton bound, so $n-2 d+2=k \geq 0$; hence, $n \geq 2 d-2$. Therefore, a nontrivial quantum MDS code satisfies $n \geq 2 d-2 \geq 4$.

By Lemma 61] the existence of an $[[n, n-2 d+2, d]]_{q}$ stabilizer code implies the existence of classical MDS codes $C$ and $C^{\perp_{a}}$ with parameters $[n, d-1, n-d+2]_{q^{2}}$ and $[n, n-d+1, d]_{q^{2}}$, respectively. If the quantum code is a nontrivial MDS code, then the associated classical codes are nontrivial classical MDS codes. Indeed, for $n \geq 4$ the quantum Singleton bound implies $d \leq(n+2) / 2 \leq(2 n-2) / 2=n-1$, so $C$ is a nontrivial classical MDS code.

By Lemma 62, the dimension of $C$ satisfies the constraints $2 \leq d-1 \leq$ $\min \left\{n-2, q^{2}-1\right\}$, or equivalently $3 \leq d \leq \min \left\{n-1, q^{2}\right\}$. Similarly, the length $n$ of $C$ satisfies $n \leq q^{2}+(d-1)-1 \leq 2 q^{2}-2$. If we combine these inequalities then we get our claim. 
Example 64. The length of a nontrivial binary MDS stabilizer code cannot exceed $2 q^{2}-2=6$. In [19] the nontrivial MDS stabilizer codes for $q=2$ were found to be $[[5,1,3]]_{2}$ and $[[6,0,4]]_{2}$, so there cannot exist further nontrivial MDS stabilizer codes.

In [47], the question of the maximal length of MDS codes was raised. All MDS stabilizer codes provided in that reference had a length of $q^{2}$ or less; this prompted us to look at the following famous conjecture for classical codes (cf. [52, Theorem 7.4.5] or [67, pages 327-328]).

MDS Conjecture. If there is a nontrivial $[n, k]_{q}$ MDS code, then $n \leq q+1$ except when $q$ is even and $k=3$ or $k=q-1$ in which case $n \leq q+2$.

If the MDS conjecture is true (and much supporting evidence is known), then we can improve upon the result of Theorem 63.

Corollary 65. If the classical MDS conjecture holds, then there are no nontrivial MDS stabilizer codes of lengths exceeding $q^{2}+1$ except when $q$ is even and $d=4$ or $d=q^{2}$ in which case $n \leq q^{2}+2$.

\section{Quantum Character Codes}

A new family of codes was introduced in [32]. The codes of this family are defined using group characters. These codes are in many ways remarkably similar to binary Reed-Muller codes, but they are defined over nonbinary fields. Since these codes were introduced only recently and are not yet wellknown, we will provide a little more background. In this section we derive quantum codes from group character codes using the CSS construction.

Group character codes. Let $G$ be an additive abelian group of order $n$ and exponent $m$. Let $\mathbf{F}_{q}$ be a finite field such that $\operatorname{gcd}(n, q)=1$ and $m \mid q-1$.

The set $\operatorname{Hom}\left(G, \mathbf{F}_{q}^{*}\right)$ of $\mathbf{F}_{q}$-valued characters of $G$ consists of the homomorphisms from $G$ into the multiplicative group $\mathbf{F}_{q}^{*}$. Our assumptions ensure that the set of characters forms a group that is isomorphic to $G$. We can index the characters by elements of the group $G$,

$$
\operatorname{Hom}\left(G, \mathbf{F}_{q}^{*}\right)=\left\{\chi_{x} \mid x \in G\right\},
$$

such that $\chi_{0}$ denotes the trivial character, and $\chi_{-x}$ denotes the inverse of $\chi_{x}$.

For any subset $X$ of the group $G$, the character code $C_{X}$ is defined as

$$
C_{X}=\left\{c \in \mathbf{F}_{q}^{n} \mid \sum_{i=0}^{n-1} c_{i} \chi_{x_{i}}(y)=0 \text { for all } y \in X\right\} .
$$


The code $C_{X}$ is an $[n, k]_{q}$ code with $n=|G|$ and $k=n-|X|$. The parity check matrix $H_{X}$ of the code $C_{X}$, with $X=\left\{x_{0}, \ldots, x_{n-k+1}\right\}$, is given by

$$
H_{X}=\left(\begin{array}{cccc}
\chi_{x_{0}}\left(x_{0}\right) & \chi_{x_{1}}\left(x_{0}\right) & \cdots & \chi_{x_{n-1}}\left(x_{0}\right) \\
\chi_{x_{0}}\left(x_{1}\right) & \chi_{x_{1}}\left(x_{1}\right) & \cdots & \chi_{x_{n-1}}\left(x_{1}\right) \\
\vdots & \vdots & \ddots & \vdots \\
\chi_{x_{0}}\left(x_{n-k-1}\right) & \chi_{x_{1}}\left(x_{n-k-1}\right) & \cdots & \chi_{x_{n-1}}\left(x_{n-k-1}\right)
\end{array}\right)
$$

and its generator matrix $G_{X}$ by

$$
G_{X}=\left(\begin{array}{cccc}
\chi_{x_{0}}\left(-x_{n-k}\right) & \chi_{x_{1}}\left(-x_{n-k}\right) & \cdots & \chi_{x_{n-1}}\left(-x_{n-k}\right) \\
\chi_{x_{0}}\left(-x_{n-k+1}\right) & \chi_{x_{1}}\left(-x_{n-k+1}\right) & \cdots & \chi_{x_{n-1}}\left(-x_{n-k+1}\right) \\
\vdots & \vdots & \ddots & \vdots \\
\chi_{x_{0}}\left(-x_{n-1}\right) & \chi_{x_{1}}\left(-x_{n-1}\right) & \cdots & \chi_{x_{n-1}}\left(-x_{n-1}\right)
\end{array}\right)
$$

Indeed, the characters satisfy the well-known orthogonality relation

$$
\sum_{x \in G} \chi_{x}(y) \chi_{x}(z)= \begin{cases}n & \text { if } y+z=\mathbf{0} \\ 0 & \text { if } y+z \neq \mathbf{0}\end{cases}
$$

which implies $G_{X} H_{X}^{T}=0$.

Elementary abelian 2-groups. We now specialize to the case of a finite elementary abelian 2-group $G=\mathbf{Z}_{2}^{m}, m \geq 1$. Let $\mathbf{F}_{q}$ be a finite field of odd characteristic; this choice ensures that $2 \mid q-1$ and $\operatorname{gcd}\left(2^{m}, q\right)=1$. Recall that the characters of $G$ are given by $\chi_{x}(y)=(-1)^{x \cdot y}$ for $x, y$ in $G$.

We define a 2 -group character $\operatorname{code} \mathcal{C}_{q}(r, m)$ by

$$
\mathcal{C}_{q}(r, m)=C_{X} \quad \text { with } \quad X=\left\{x \in \mathbf{Z}_{2}^{m} \mid \operatorname{wt}(x)>r\right\} .
$$

It can be shown that $C_{q}(r, m)$ is an $[n, k(r), d(r)]_{q}$ code, with

$$
k(r)=\sum_{j=0}^{r}\left(\begin{array}{c}
m \\
j
\end{array}\right) \quad \text { and } \quad d(r)=2^{m-r},
$$

see [32, Lemma 4 and Theorem 6]. We need the following result about 2-group character codes which is not explicitly proved in [32].

Lemma 66. If $r_{1} \leq r_{2}$, then $\mathcal{C}_{q}\left(r_{1}, m\right) \subseteq \mathcal{C}_{q}\left(r_{2}, m\right)$. 
Proof. By equation (23) the generator matrix of $\mathcal{C}_{q}(r, m)$ consists of vectors of the form

$$
\left(\chi_{x_{0}}\left(x_{i}\right), \chi_{x_{1}}\left(x_{i}\right), \cdots, \chi_{x_{n-1}}\left(x_{i}\right)\right)=\left(\chi_{x_{0}}\left(-x_{i}\right), \chi_{x_{1}}\left(-x_{i}\right), \cdots, \chi_{x_{n-1}}\left(-x_{i}\right)\right)
$$

where $x_{i}$ is an element of $\mathbf{Z}_{2}^{m}$ of Hamming weight wt $\left(x_{i}\right) \leq r$. Thus, the generator matrix of $C_{q}\left(r_{1}, m\right)$ is a submatrix of the generator matrix of $C_{q}\left(r_{2}, m\right)$, which shows that $\mathcal{C}_{q}\left(r_{1}, m\right) \subseteq \mathcal{C}_{q}\left(r_{2}, m\right)$.

Lemma 67. The dual code $\mathcal{C}_{q}(r, m)^{\perp}$ is equivalent to $\mathcal{C}_{q}(m-r-1, m)$.

Proof. See [32, Theorem 8].

Now we will construct a family of codes based on the CSS construction.

Theorem 68. If $0 \leq r_{1}<r_{2} \leq m$ and $q$ the power of an odd prime, then there exists an $\left[\left[n, k\left(r_{2}\right)-k\left(r_{1}\right), \min \left\{2^{m-r_{2}}, 2^{r_{1}+1}\right\}\right]\right]_{q}$ quantum code, where $n=2^{m}$ and $k(r)$ is given by equation 24).

Proof. If $r_{1}<r_{2}$, then $C_{1}=\mathcal{C}_{q}\left(r_{1}, m\right) \subseteq \mathcal{C}_{q}\left(r_{2}, m\right)=C_{2}$ by Lemma 66. From the equations for the minimum distances given in (24), we can see that wt $\left(C_{2} \backslash C_{1}\right)=2^{m-r_{2}}$. Similarly, it follows from Lemma 67 that $\operatorname{wt}\left(C_{1}^{\perp} \backslash\right.$ $\left.C_{2}^{\perp}\right)=\operatorname{wt}\left(\mathcal{C}_{q}\left(m-r_{1}-1\right) \backslash \mathcal{C}_{q}\left(m-r_{2}-1\right)\right)=2^{r_{1}+1}$. By Lemma 20, there exists an $\left[\left[n, k\left(r_{2}\right)-k\left(r_{1}\right), \min \left\{2^{m-r_{2}}, 2^{r_{1}+1}\right\}\right]\right]_{q}$ stabilizer code, where the dimensions $k\left(r_{1}\right)$ and $k\left(r_{2}\right)$ are given by equation (24).

We can get more quantum codes by puncturing, as we did in the case of $\mathrm{BCH}$ codes. However, only the weight distribution of $\mathcal{C}_{q}(1, m)$ is known, so at the moment we do not have enough information as to what codes might exist.

\section{Code Constructions}

Constructing good quantum codes is a difficult task. We need a quantum code for each parameter $n$ and $k$ in our tables. We collect in this section some simple facts about the construction of codes. Lemmas 69 [71 show how to lengthen, shorten or reduce the dimension of the stabilizer code.

Lemma 69. If an $[[n, k, d]]_{q}$ stabilizer code exists for $k>0$, then there exists an impure $[[n+1, k, d]]_{q}$ stabilizer code. 


\begin{tabular}{c||c|c|c}
$\mathrm{n} / \mathrm{k}$ & $k-1$ & $k$ & $k+1$ \\
\hline \hline$n-1$ & $\begin{array}{c}d-1 \text { pure } \\
\text { Lemma[7] }\end{array}$ & $\begin{array}{c}d-1 \text { pure } \\
\text { Lemma[7] }\end{array}$ & $\begin{array}{c}d-1 \text { pure } \\
\text { Lemma [0] }\end{array}$ \\
\hline$n$ & $\begin{array}{c}d \text { pure } \\
\text { Lemma[7] }\end{array}$ & $d$ pure & \\
\hline$n+1$ & $\begin{array}{c}d \text { impure } \\
\text { Lemma[69] }\end{array}$ & $\begin{array}{c}d \text { impure } \\
\text { Lemma[69] }\end{array}$ &
\end{tabular}

Table 1: The existence of a pure $[[n, k, d]]_{q}$ stabilizer code implies the existence of codes with other parameters.

Proof. If an $[[n, k, d]]_{q}$ stabilizer code exists, then there exists an additive subcode $C \leq \mathbf{F}_{q}^{2 n}$ such that $|C|=q^{n-k}, C \leq C^{\perp_{s}}$, and $\operatorname{swt}\left(C^{\perp_{s}} \backslash C\right)=d$. Define the additive code

$$
C^{\prime}=\left\{(a \alpha \mid b 0) \mid \alpha \in \mathbf{F}_{q},(a \mid b) \in \mathbf{F}_{q}^{2 n}\right\} .
$$

We have $\left|C^{\prime}\right|=q^{n-k+1}$. The definition ensures that $C^{\prime}$ is self-orthogonal with respect to the trace-symplectic inner product. Indeed, two arbitrary elements $(a \alpha \mid b 0)$ and $\left(a^{\prime} \alpha^{\prime} \mid b^{\prime} 0\right)$ of $C^{\prime}$ satisfy the orthogonality condition

$$
\left\langle(a \alpha \mid b 0) \mid\left(a^{\prime} \alpha^{\prime} \mid b^{\prime} 0\right)\right\rangle_{s}=\left\langle(a \mid b) \mid\left(a^{\prime} \mid b^{\prime}\right)\right\rangle_{s}+\operatorname{tr}\left(\alpha \cdot 0-\alpha^{\prime} \cdot 0\right)=0 .
$$

A vector in the trace-symplectic dual of $C^{\prime}$ has to be of the form $(a \alpha \mid b 0)$ with $(a \mid b) \in C^{\perp_{s}}$ and $\alpha \in \mathbf{F}_{q}$. Furthermore,

$$
\operatorname{swt}\left(C^{\perp_{s}} \backslash C^{\prime}\right)=\min \left\{\operatorname{swt}(a \alpha \mid b 0) \mid \alpha \in \mathbf{F}_{q}, a, b \in C^{\perp_{s}} \backslash C\right\},
$$

which coincides with $\operatorname{swt}\left(C^{\perp_{s}} \backslash C\right)$. Therefore, an $[[n+1, k, d]]_{q}$ stabilizer code exists by Theorem [13. If $d>1$, then the code is impure, because $C^{\prime \perp_{s}}$ contains the vector $(0 \alpha \mid 00)$ of symplectic weight 1 .

Lemma 70. If a pure $[[n, k, d]]_{q}$ stabilizer code exists with $n \geq 2$ and $d \geq 2$, then there exists a pure $[[n-1, k+1, d-1]]_{q}$ stabilizer code.

Proof. If a pure $[[n, k, d]]_{q}$ stabilizer code exists, then there exists an additive code $D \leq \mathbf{F}_{q^{2}}^{n}$ that is self-orthogonal with respect to the trace-alternating form, so that $|D|=q^{n-k}$ and $\operatorname{wt}\left(D^{\perp_{a}}\right)=d$. Let $D_{0}^{\perp_{a}}$ denote the code obtained by puncturing the first coordinate of $D^{\perp_{a}}$. Since the minimum distance of $D^{\perp_{a}}$ is at least 2 , we know that $\left|D_{0}^{\perp_{a}}\right|=\left|D^{\perp_{a}}\right|=q^{n+k}$, and we note that the minimum distance of $D_{0}^{\perp_{a}}$ is $d-1$. The dual of $D_{0}^{\perp_{a}}$ consists of all vectors $u$ in $\mathbf{F}_{q^{2}}^{n-1}$ such that $0 u$ is contained in $D$. Furthermore, if $u$ is 
an element of $D_{0}$, then $0 u$ is contained in $D$; hence, $D_{0}$ is a self-orthogonal additive code. The code $D_{0}$ is of size $q^{(n-1)-(k+1)}$, because

$$
\operatorname{dim} D_{0}+\operatorname{dim} D_{0}^{\perp_{a}}=\operatorname{dim} \mathbf{F}_{q^{2}}^{n-1}
$$

when we view $D_{0}$ and its dual as $\mathbf{F}_{p}$-vector spaces. It follows that there exists a pure $[[n-1, k+1, d-1]]_{q}$ stabilizer code.

Lemma 71. If a (pure) $[[n, k, d]]_{q}$ stabilizer code exists, with $k \geq 2(k \geq 1)$, then there exists a (pure) $\left[\left[n, k-1, d^{*}\right]\right]_{q}$ stabilizer code such that $d^{*} \geq d$.

Proof. If an $[[n, k, d]]_{q}$ stabilizer code exists, then there exists an additive code $D \leq \mathbf{F}_{q^{2}}^{n}$ such that $D \leq D^{\perp_{a}}$ with $\operatorname{wt}\left(D^{\perp_{a}} \backslash D\right)=d$ and $|D|=q^{n-k}$. Choose an additive code $D_{b}$ of size $\left|D_{b}\right|=q^{n-k+1}$ such that $D \leq D_{b} \leq D^{\perp_{a}}$. Since $D \leq D_{b}$, we have $D_{b}^{\perp_{a}} \leq D^{\perp_{a}}$. The set $\Sigma_{b}=D_{b}^{\perp_{a}} \backslash D_{b}$ is a subset of $D^{\perp_{a}} \backslash D$, hence the minimum weight $d^{*}$ of $\Sigma_{b}$ is at least $d$. This proves the existence of an $\left[\left[n, k-1, d^{*}\right]\right]$ code.

If the code is pure, then $\operatorname{wt}\left(D^{\perp_{a}}\right)=d$; it follows from $D_{b}^{\perp_{a}} \leq D^{\perp_{a}}$ that $\operatorname{wt}\left(D_{b}^{\perp_{a}}\right) \geq d$, so the smaller code is pure as well.

Corollary 72. If a pure $[[n, k, d]]_{q}$ stabilizer code with $n \geq 2$ and $d \geq 2$ exists, then there exists a pure $[[n-1, k, d-1]]_{q}$ stabilizer code.

Proof. Combine Lemmas [70] and [7]

Lemma 73. Suppose that an $((n, K, d))_{q}$ and an $\left(\left(n^{\prime}, K^{\prime}, d^{\prime}\right)\right)_{q}$ stabilizer code exists. Then there exists an $\left(\left(n+n^{\prime}, K K^{\prime}, \min \left(d, d^{\prime}\right)\right)_{q}\right.$ stabilizer code.

Proof. Suppose that $P$ and $P^{\prime}$ are the orthogonal projectors onto the stabilizer codes for the $((n, K, d))_{q}$ and $\left(\left(n^{\prime}, K^{\prime}, d^{\prime}\right)\right)_{q}$ stabilizer codes, respectively. Then $P \otimes P^{\prime}$ is an orthogonal projector onto a $K K^{\prime}$-dimensional subspace $Q^{*}$ of $\mathbf{C}^{d}$, where $d=q^{n+n^{\prime}}$. Let $S$ and $S^{\prime}$ respectively denote the stabilizer groups of the images of $P$ and $P^{\prime}$. Then $S^{*}=\left\{E \otimes E^{\prime} \mid E \in S, E^{\prime} \in S^{\prime}\right\}$ is the stabilizer group of $Q^{*}$.

If an element $F \otimes F^{*}$ of $G_{n} \otimes G_{n^{\prime}}=G_{n+n^{\prime}}$ is not detectable, then $F$ has to commute with all elements in $S$, and $F^{\prime}$ has to commute with all elements in $S^{\prime}$. It is not possible that both $F \in Z\left(G_{n}\right) S$ and $F^{\prime} \in Z\left(G_{n^{\prime}}\right) S^{\prime}$ hold, because this would imply that $F \otimes F^{\prime}$ is detectable. Therefore, either $F$ or $F^{\prime}$ is not detectable, which shows that the weight of $F \otimes F^{\prime}$ is at least $\min \left(d, d^{\prime}\right)$. 
Lemma 74. Let $Q_{1}$ and $Q_{2}$ be pure stabilizer codes that respectively have parameters $\left[\left[n, k_{1}, d_{1}\right]\right]_{q}$ and $\left[\left[n, k_{2}, d_{2}\right]\right]$. If $Q_{2} \subseteq Q_{1}$, then there exists a $\left[\left[2 n, k_{1}+k_{2}, d\right]\right]_{q}$ pure stabilizer code with minimum distance $d \geq \min \left\{2 d_{2}, d_{1}\right\}$.

Proof. The hypothesis implies that there exist additive subcodes $D_{1} \leq D_{2}$ of $\mathbf{F}_{q^{2}}^{n}$ such that $D_{m} \leq D_{m}^{\perp_{a}},\left|D_{m}\right|=q^{n-k_{m}}$, and $\operatorname{wt}\left(D_{m}^{\perp_{a}}\right)=d_{m}$ for $m=1,2$. The additive code

$$
D=\left\{(u, u+v) \mid u \in D_{1}, v \in D_{2}\right\} \leq \mathbf{F}_{q^{2}}^{2 n}
$$

is of size $|D|=q^{2 n-\left(k_{1}+k_{2}\right)}$. The trace-alternating dual of the code $D$ is $D^{\perp_{a}}=\left\{\left(u^{\prime}+v^{\prime}, v^{\prime}\right) \mid u^{\prime} \in D_{1}^{\perp_{a}}, v^{\prime} \in D_{2}^{\perp_{a}}\right\}$. Indeed, the vectors on the right hand side are perpendicular to the vectors in $D$, because

$$
\left\langle(u, u+v) \mid\left(u^{\prime}+v^{\prime}, v^{\prime}\right)\right\rangle_{a}=\left\langle u \mid u^{\prime}+v^{\prime}\right\rangle_{a}+\left\langle u+v \mid v^{\prime}\right\rangle_{a}=0
$$

holds for all $u \in D_{1}, v \in D_{2}$ and $u^{\prime} \in D_{1}^{\perp_{a}}, v^{\prime} \in D_{2}^{\perp_{a}}$. We observe that $D$ is self-orthogonal, $D \leq D^{\perp_{a}}$. The weight of a vector $\left(u^{\prime}+v^{\prime}, v^{\prime}\right) \in D^{\perp_{a}} \backslash D$ is at least $\min \left\{2 d_{2}, d_{1}\right\}$; the claim follows.

Lemma 75 . Let $q$ be an even prime power. If a pure $\left[\left[n, k_{1}, d_{1}\right]\right]_{q}$ stabilizer code $Q_{1}$ exists that has a pure subcode $Q_{2} \subseteq Q_{1}$ with parameters $\left[\left[n, k_{2}, d_{2}\right]\right]_{q}$ such that $k_{1}>k_{2}$, then a pure $\left[\left[2 n, k_{1}-k_{2}, d\right]\right]_{q}$ stabilizer code exists such that $d \geq \min \left\{2 d_{1}, d_{2}\right\}$.

Proof. If an $\left[\left[n_{m}, k_{m}, d_{m}\right]\right]_{q}$ stabilizer code exists, then there exists an additive code $D_{m} \leq \mathbf{F}_{q^{2}}^{n}$ such that $D_{m} \leq D_{m}^{\perp_{a}}$, wt $\left(D_{m}^{\perp_{a}}\right)=d$, and $\left|D_{m}\right|=q^{n-k_{m}}$ for $m=1,2$. The inclusion $Q_{2} \subseteq Q_{1}$ implies that $D_{1} \leq D_{2}$. Let $D$ denote the additive code consisting of vectors of the form $(u, u+v)$ such that $u \in D_{2}^{\perp a}$ and $v \in D_{1}$.

We claim that $D^{\perp_{a}}$ consists of vectors of the form $\left(u^{\prime}, u^{\prime}+v^{\prime}\right)$ such that $u^{\prime} \in D_{1}^{\perp_{a}}$ and $v^{\prime} \in D_{2}$. Indeed, let $v_{1}=(u, u+v)$ denote a vector in $D$, and let $v_{2}=\left(u^{\prime}, u^{\prime}+v^{\prime}\right)$ be a vector with $u^{\prime} \in D_{1}^{\perp_{a}}$ and $v^{\prime} \in D_{2}$. We have

$$
\left\langle v_{1} \mid v_{2}\right\rangle_{a}=\left\langle u \mid u^{\prime}\right\rangle_{a}+\left\langle u \mid u^{\prime}\right\rangle_{a}+\left\langle u \mid v^{\prime}\right\rangle_{a}+\left\langle v \mid u^{\prime}\right\rangle_{a}+\left\langle v \mid v^{\prime}\right\rangle_{a} .
$$

The first two terms on the right hand side cancel because the characteristic of the field is even; the next two terms vanish since the vectors belong to dual spaces; the last term vanishes because $v$ and $v^{\prime}$ are both contained in $D_{2}$, and $D_{2}$ is self-orthogonal. Therefore, $v_{1}$ and $v_{2}$ are orthogonal. The set $\left\{\left(u^{\prime}, u^{\prime}+v^{\prime}\right) \mid u^{\prime} \in D_{1}^{\perp_{a}}, v^{\prime} \in D_{2}\right\} \subseteq D^{\perp_{a}}$ has cardinality $q^{2 n+k_{1}-k_{2}}$, so it must be equal to $D^{\perp_{a}}$ by a dimension argument.

The Hamming weight of a vector $\left(u^{\prime}, u^{\prime}+v^{\prime}\right)$ in $D^{\perp_{a}}$ is at least $\min \left\{2 d_{1}, d_{2}\right\}$, because $u^{\prime} \in D_{1}^{\perp_{a}}$ and $v^{\prime} \in D_{2} \leq D_{2}^{\perp_{a}}$. 
Lemma 76. Let $q$ be a power of a prime. If an $((n, K, d))_{q^{m}}$ stabilizer code exists, then an $((n m, K, \geq d))_{q}$ stabilizer code exists. Conversely, if an $((n m, K, d))_{q}$ stabilizer code exists, then there exists an $((n, K, \geq\lfloor d / m\rfloor))_{q^{m}}$ stabilizer code.

This lemma is implicitly contained in the paper by Ashikhmin and Knill [4].

Proof. Let $B=\left\{\beta_{1}, \ldots, \beta_{m}\right\}$ denote a basis of $\mathbf{F}_{q^{m}} / \mathbf{F}_{q}$. A nondegenerate symmetric form on the $\mathbf{F}_{q^{-}}$-vector space $\mathbf{F}_{q^{m}}$ is given by $\operatorname{tr}_{q^{m} / q}(x y)$. It follows that the Gram matrix $M=\left(\operatorname{tr}_{q^{m} / q}\left(\beta_{i} \beta_{j}\right)\right)_{1 \leq i, j \leq m}$ is nonsingular. We have $\operatorname{tr}_{q^{m} / q}(x y)=e_{B}(x)^{t} M e_{B}(y)$ for all $x, y$ in $\mathbf{F}_{q^{m}}$.

If $a$ is an element of $\mathbf{F}_{q^{m}}$, then we denote by $e_{B}(a)$ the coordinate vector in $\mathbf{F}_{q}^{m}$ given by $e_{B}(a)=\left(a_{1}, \ldots, a_{m}\right)$, where $a=\sum_{i=1}^{m} a_{i} \beta_{i}$. We define an $\mathbf{F}_{p}$-vector space isomorphism $\varphi_{B}$ from $\mathbf{F}_{q^{m}}^{2 n}$ onto $\mathbf{F}_{q}^{2 n m}$ by

$$
\varphi_{B}((a \mid b))=\left(\left(e_{B}\left(a_{1}\right), \ldots, e_{B}\left(a_{n}\right)\right) \mid\left(M e_{B}\left(b_{1}\right), \ldots, M e_{B}\left(b_{n}\right)\right)\right) .
$$

It follows from the fact that $\operatorname{tr}_{q^{m} / q}\left(\operatorname{tr}_{q / p}(x)\right)=\operatorname{tr}_{q^{m} / p}(x)$ holds for all $x$ in $\mathbf{F}_{q^{m}}$ and the definition of the isomorphism $\varphi_{B}$ that $(a \mid b) \perp_{s}(c \mid d)$ holds in $\mathbf{F}_{q^{m}}^{2 n}$ if and only if $\varphi_{B}((a \mid b)) \perp_{s} \varphi_{B}((c \mid d))$ holds in $\mathbf{F}_{q^{2 n m}}$.

If an $((n, K, d))_{q^{m}}$ exists, then there exists an additive code $C \leq \mathbf{F}_{q^{m}}^{2 n}$ of size $|C|=q^{n m} / K$ such that $C \leq C^{\perp_{s}}, \operatorname{swt}\left(C^{\perp_{s}} \backslash C\right)=d$ if $K>1$, and $\operatorname{swt}\left(C^{\perp_{s}}\right)=d$ if $K=1$. Therefore, the code $\varphi_{B}(C)$ over the alphabet $\mathbf{F}_{q}$ is of size $q^{n m} / K$, satisfies $\varphi_{B}(C) \leq \varphi_{B}(C)^{\perp_{s}} \leq \mathbf{F}_{q}^{2 n m}$, and $\operatorname{swt}\left(\varphi_{B}(C)^{\perp_{s}} \backslash \varphi_{B}(C)\right)=d$ if $K>1$ and $\operatorname{swt}\left(\varphi_{B}(C)^{\perp_{s}}\right)=d$ if $K=1$. Thus, an $((n m, K, d))_{q}$ stabilizer code exists.

The existence of an $((n m, K, d))_{q}$ stabilizer code implies the existence of an $((n m, K))_{q}$ stabilizer code; the claim about the minimum distance follows from the fact that $\varphi_{B}^{-1}$ maps each nonzero block of $m$ symbols to a nonzero symbol in $\mathbf{F}_{q^{m}}$.

We notice that if $q$ is even or if $q$ and $m$ are both odd, then there exists a basis $B$ such that $M$ is the identity matrix; in that case, $\varphi_{B}$ simply expands each symbol into coordinates with respect to $B$. If $q$ is odd and $m$ is even, then no such basis exists.

\section{Conclusions and Open Problems}

We have further developed the theory of nonbinary stabilizer codes. In the first seven sections, we studied the basic theory of nonbinary stabilizer codes 
over finite fields, and introduced Galois-theoretic methods to clarify the relation between these and more general quantum codes. In the remaining sections, we derived numerous families of quantum codes. Table 2 gives an overview and summarizes the main parameters of these families.

We should emphasize that it is possible to start with a different choice of error basis [60], and one can develop a similar theory for such stabilizer codes. For example, one choice leads to self-orthogonal additive subcodes of $\mathbf{Z}_{q}^{n} \times \mathbf{Z}_{q}^{n}$ instead of subcodes of $\mathbf{F}_{q}^{n} \times \mathbf{F}_{q}^{n}$. It would be interesting to know how the stabilizer codes with respect to different error bases compare.

One central theme in quantum error-correction is the construction of codes that have a large minimum distance. We were able to show that the length of an MDS stabilizer code over $\mathbf{F}_{q}$ cannot exceed $q^{2}+1$, except in a few sporadic cases, assuming that the classical MDS conjecture holds. An open problem is whether the length $n$ of a $q$-ary quantum MDS code is bounded by $q^{2}+1$ for all but finitely many $n$.

A number of researchers raised the question whether there exist degenerate quantum codes that can exceed the quantum Hamming bound. Following Gottesman's lead [40], we were able to show that single and double error-correcting nonbinary stabilizer codes cannot beat the quantum Hamming bound. We conjecture that no quantum error-correcting code can exceed the quantum Hamming bound, but a proof is still elusive.

Finally, we briefly mention some of the topics that we have deliberately omitted. We decided not to include tables of the best known stabilizer codes, but rather make such tables available on the home page of the second author. We selected code families that are easily accessible by elementary methods; the interested reader can find examples of more intricate algebro-geometric constructions in $[6,25,26,56,69]$ and of binary quantum LDPC codes in [21, $65,76]$. We did not include constructive aspects of encoding and decoding circuits, since encoding circuits are discussed in [49] and little is known about the decoding of stabilizer codes. We did not include combinatorial aspects, but Kim pointed out that there is a forthcoming book by Glynn, Gulliver, Maks, and Gupta that explores the relation between binary stabilizer codes and finite geometry.

Acknowledgments. We received numerous comments and suggestions during the preparation of this manuscript that are much appreciated. In particular, many thanks to Markus Grassl and Martin Rötteler for sending us corrections and suggestions, to Daniel Gottesman, Jon-Lark Kim and Simon Litsyn for sending us helpful comments and references, and to Raymond 
Laflamme and Peter Shor for providing us with historical background. We are grateful to Neil Sloane for very fruitful discussions on MDS codes, and to Gordon Chen, Phil Hemmer, and Suhail Zubairy for helpful discussions in our quantum computing seminar.

This work would not have been possible without the support of NSF grant CCR-0218582, NSF CAREER award CCF-0347310, a TITF grant, and a TEES Select Young Faculty Award. 


\begin{tabular}{|c|c|c|c|}
\hline Family & {$[[n, k, d]]_{q}$} & Purity & Parameter Ranges and References \\
\hline$\overline{\overline{\text { Short MDS }}}$ & $\overline{[n, n-2 d+2, d]]_{q}}$ & pure & $2 \leq d \leq\lceil n / 2\rceil, q^{2}-1 \geq\left(\begin{array}{l}n \\
d\end{array}\right)$ \\
\hline Hermitian Hamming & $\overline{[n, n-2 m, 3]]_{q}}$ & pure & $m \geq 2, \operatorname{gcd}\left(m, q^{2}-1\right)=1, n=\left(q^{2 m}-1\right) /\left(q^{2}-1\right)$ \\
\hline Euclidean Hamming & $[n, n-2 m, 3]]_{q}$ & pure & $m \geq 2, \operatorname{gcd}(m, q-1)=1, n=\left(q^{m}-1\right) /(q-1)$ \\
\hline Quadratic Residue I & {$[[n, 1, d]]_{q}$} & pure & $\begin{array}{c}n \text { prime, } n \equiv 3 \bmod 4, q \not \equiv 0 \bmod n \\
q \text { is a quadratic residue modulo } n, d^{2}-d+1 \geq n\end{array}$ \\
\hline Quadratic Residue II & {$[[n, 1, d]]_{q}$} & pure & $\begin{array}{c}n \text { prime, } n \equiv 1 \bmod 4, q \not \equiv 0 \bmod n \\
q \text { is a quadratic residue modulo } n, d \geq \sqrt{n}\end{array}$ \\
\hline Melas & $[n, n-4 m, \geq 3]]_{q}$ & pure & $q$ even, $n=q^{2 m}-1$, Pure to 3 \\
\hline 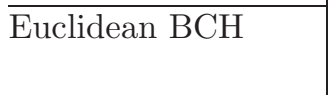 & {$[[n, n-2 m\lceil(\delta-1)(1-1 / q)], \geq \delta]]_{q}$} & $\begin{array}{l}\text { pure } \\
\text { to } \delta\end{array}$ & $\begin{array}{c}2 \leq \delta \leq q^{|m / 2|}-1-(q-2)[m \text { odd }] \\
n=q^{m}-1 \text { and } m \geq 2\end{array}$ \\
\hline Punctured BCH & {$\left[\left[d^{*}(\mu), \geq d^{*}(\mu)-2 m\lceil(\delta-1)(1-1 / q)], \geq \delta\right]\right]_{q}$} & pure? & $\delta<q^{\lfloor m / 2\rceil}-1$, See Corollary 58 \\
\hline $\begin{array}{l}\text { Hermitian } \mathrm{BCH} \\
\text { Extended } \mathrm{BCH}\end{array}$ & $\begin{array}{c}{\left[\left[n, n-2 m\left\lceil(\delta-1)\left(1-1 / q^{2}\right)\right], \geq \delta\right]\right]_{q}} \\
{\left[\left[n+1, n-2 m\left\lceil(\delta-1)\left(1-1 / q^{2}\right)\right]-1, \geq \delta+1\right]\right]_{q}}\end{array}$ & $\begin{array}{l}\text { pure } \\
\text { pure }\end{array}$ & $\begin{array}{c}2 \leq \delta \leq q^{m}-1, n=q^{2 m}-1, \text { Pure to } \delta \\
\text { Pure to } \delta+1\end{array}$ \\
\hline Trivial MDS & $\begin{array}{c}{[[n, n-2,2]]_{q}} \\
{[[n, n, 1]]_{q}} \\
\end{array}$ & $\begin{array}{l}\text { pure } \\
\text { pure } \\
\end{array}$ & $\begin{array}{c}n \equiv 0 \bmod p \\
n \geq 1\end{array}$ \\
\hline Character & $\left.\left[n, k\left(r_{2}\right)-k\left(r_{1}\right), \min \left\{2^{m-r_{2}}, 2^{r_{1}+1}\right\}\right]\right]_{q}$ & pure & $n=2^{m}, q$ odd $, 0 \leq r_{1}<r_{2} \leq m, k(r)=\sum_{j=0}^{r}\left(\begin{array}{c}m \\
j\end{array}\right)$ \\
\hline $\begin{array}{l}\text { CSS GRM } \\
\text { Punctured GRM }\end{array}$ & $\begin{array}{c}{\left[\left[q^{m}, k\left(\nu_{2}\right)-k\left(\nu_{1}\right), \min \left\{d\left(\nu_{2}\right), d\left(\nu_{1}^{\perp}\right)\right\}\right]\right]_{q}} \\
0 \leq \nu_{1} \leq \nu_{2} \leq m(q-1)-1 \\
{\left[\left[d(\mu), \geq k\left(\nu_{2}\right)-k\left(\nu_{1}\right)-(n-d(\mu)), \geq d\right]\right]_{q}}\end{array}$ & $\begin{array}{l}\text { pure } \\
\text { pure? }\end{array}$ & $\begin{array}{c}k(\nu)=\sum_{j=0}^{m}(-1)^{j}\left(\begin{array}{c}m \\
j\end{array}\right)\left(\begin{array}{c}m+\nu-j q \\
\nu-j q\end{array}\right), \nu^{\perp}=m(q-1)-\nu-1 \\
\nu^{\perp}+1=(q-1) Q+R, d(\nu)=(R+1) q^{Q} \\
d \geq \min \left\{d\left(\nu_{2}\right), d\left(\nu_{1}^{\perp}\right), 0 \leq \mu \leq \nu_{2}-\nu_{1} ;[84]\right.\end{array}$ \\
\hline$\overline{\text { Hermitian GRM }}$ & $\begin{array}{c}{\left[\left[q^{2 m}, q^{2 m}-2 k(\nu), d\left(\nu^{\perp}\right)\right]\right]_{q}} \\
0 \leq \nu \leq m(q-1)-1 \\
{\left[\left[d\left(\mu^{\perp}\right), \geq d\left(\mu^{\perp}\right)-2 k(\nu), \geq d\left(\nu^{\perp}\right)\right]\right]_{q}}\end{array}$ & $\begin{array}{l}\text { pure } \\
\text { pure? }\end{array}$ & $\begin{array}{c}k(\nu)=\sum_{j=0}^{m}(-1)^{j}\left(\begin{array}{c}m \\
j\end{array}\right)\left(\begin{array}{c}m+\nu-j q^{2} \\
\nu-j q^{2}\end{array}\right), \nu^{\perp}=m\left(q^{2}-1\right)-\nu-1 \\
\nu^{\perp}+1=\left(q^{2}-1\right) Q+R, d(\nu)=(R+1) q^{2 Q} \\
(\nu+1) q \leq \mu \leq m\left(q^{2}-1\right)-1 ;[84]\end{array}$ \\
\hline Punctured MDS & {$\left[\left[q^{2}-q \alpha, q^{2}-q \alpha-2 \nu-2, \nu+2\right]\right]_{q}$} & pure & $0 \leq \nu \leq q-2,0 \leq \alpha \leq q-\nu-1 ;[84]$ \\
\hline Euclidean MDS & {$[[n, n-2 d+2, d]]_{q}$} & pure & $3 \leq n \leq q, 1 \leq d \leq n / 2+1 ;[49]$ \\
\hline Hermitian MDS & {$\left[\left[q^{2}-s, q^{2}-s-2 d+2, d\right]\right]_{q}$} & pure & $1 \leq d \leq q, s=0,1 ;[49]$ \\
\hline Twisted & {$\left[\left[q^{2}+1, q^{2}-3,3\right]\right]_{q}$} & pure? & [16] \\
\hline Extended Twisted & $\begin{array}{l}{\left[\left[q^{r}, q^{r}-r-2,3\right]\right]_{q}} \\
{[[n, n-r-2,3]]_{q}}\end{array}$ & $\begin{array}{l}\text { pure } \\
\text { pure }\end{array}$ & $\begin{array}{c}r \geq 2 ;[16] \\
n=\left(q^{r+2}-q^{3}\right) /\left(q^{2}-1\right), r \geq 1, r \text { odd; }[16]\end{array}$ \\
\hline$\overline{\text { Perfect }}$ & {$[[n, n-r-2,3]]_{q}$} & pure & $n=\left(q^{r+2}-1\right) /\left(q^{2}-1\right), r \geq 2, r$ even; $[16]$ \\
\hline
\end{tabular}

Table 2: A compilation of known families of quantum codes 


\section{References}

[1] D. Aharonov and M. Ben-Or. Fault-tolerant quantum computation with constant error. In Proc. of the 29th Annual ACM Symposium on Theory of Computation (STOC), pages 176-188, New York, 1997. ACM.

[2] S. A. Aly, P. K. Sarvepalli, and A. Klappenecker. On the dimension, minimum distance, and duals of primitive BCH codes. eprint quant-ph/0501126 2005.

[3] V. Arvind and K.R. Parthasarathy. A family of quantum stabilizer codes based on the Weyl commutation relations over a finite field. In A tribute to C. S. Seshadri (Chennai, 2002), Trends Math., pages 133-153. Birkhäuser, 2003.

[4] A. Ashikhmin and E. Knill. Nonbinary quantum stabilizer codes. IEEE Trans. Inform. Theory, 47(7):3065-3072, 2001.

[5] A. Ashikhmin and S. Litsyn. Upper bounds on the size of quantum codes. IEEE Trans. Inform. Theory, 45(4):1206-1215, 1999.

[6] A. Ashikhmin, M.A. Tsfasman, and S. Litsyn. Asymptotically good quantum codes. Phys. Rev. A, 63:032311, 2001.

[7] A.E. Ashikhmin, A.M. Barg, E. Knill, and S.N. Litsyn. Quantum error detection I: Statement of the problem. IEEE Trans. on Information Theory, 46(3):778-788, 2000.

[8] A.E. Ashikhmin, A.M. Barg, E. Knill, and S.N. Litsyn. Quantum error detection II: Bounds. IEEE Trans. on Information Theory, 46(3):789-800, 2000.

[9] A.R. Assmus, Jr. and J.D. Key. Designs and their codes. Cambridge University Press, Cambridge, 1992.

[10] E.F. Assmus, Jr. and J.D. Key. Polynomial codes and finite geometries. In V.S. Pless and W.C. Huffman, editors, Handbook of Coding Theory, volume II, pages 1269-1343, Amsterdam, 1998. Elsevier.

[11] A. Barg, S. Guritman, and J. Simonis. Strengthening the Gilbert-Varshamov bound. Linear Algebra and its Application, 307:119-129, 2000.

[12] H. Barnum. Quantum message authentication codes. quant-ph/0103123 version 2, 2001.

[13] H. Barnum, C. Crepeau, D. Gottesman, A. Smith, and A. Tapp. Authentication of quantum messages. In Proc. 43rdAnnual IEEE Symposium on the Foundations of Computer Science (FOCS '02), pages 449-458. IEEE Press, 2002.

[14] T. Beth and M. Grassl. The quantum Hamming and hexacodes. Fortschr. Phys., 46(45):459-491, 1998.

[15] A. Betten, H. Fripertinger, A. Kerber, A. Wassermann, and K.-H. Zimmermann. Codierungstheorie - Konstruktion und Anwendung linearer Codes. Springer-Verlag, Berlin, 1998.

[16] J. Bierbrauer and Y. Edel. Quantum twisted codes. J. Comb. Designs, 8:174-188, 2000.

[17] G. Birkhoff. Lattice Theory. AMS, 2nd edition, 1961.

[18] A.R. Calderbank, E.M. Rains, P.W. Shor, and N.J.A. Sloane. Quantum error correction and orthogonal geometry. Phys. Rev. Lett., 76:405-409, 1997.

[19] A.R. Calderbank, E.M. Rains, P.W. Shor, and N.J.A. Sloane. Quantum error correction via codes over GF(4). IEEE Trans. Inform. Theory, 44:1369-1387, 1998. 
[20] A.R. Calderbank and P. Shor. Good quantum error-correcting codes exist. Phys. Rev. A, 54:1098-1105, 1996.

[21] T. Camara, H. Ollivier, and J.-P. Tillich. Constructions and performance of classes of quantum LDPC codes. eprint: quant-ph/0502086 2005.

[22] P. Charpin. Open problems on cyclic codes. In V.S. Pless and C.W. Huffman, editors, Handbook of Coding Theory, pages 963-1063. Elsevier, 1998.

[23] H.F. Chau. Correcting quantum errors in higher spin systems. Phys. Rev. A, 55:R839-R841, 1997.

[24] H.F. Chau. Five quantum register error correction code for higher spin systems. Phys. Rev. A, 56:R1-R4, 1997.

[25] H. Chen. Some good quantum error-correcting codes from algebraic-geometric codes. IEEE Trans. Inform. Theory, 47:2059-2061, 2001.

[26] H. Chen, S. Ling, and C. Xing. Asymptotically good quantum codes exceeding the Ashikhmin-Litsyn-Tsfasman bound. IEEE Trans. Inform. Theory, 47(5):2055-2058, 2001.

[27] R. Cleve. Quantum stabilizer codes and classical linear codes. Phys. Rev. A, 55(6):4054-4059, 1997.

[28] R. Cleve and D. Gottesman. Efficient computations of encodings for quantum error correction. Phys. Rev. A, 56(1):76-82, 1997.

[29] G. Cohen, S. Encheva, and S. Litsyn. On binary constructions of quantum codes. IEEE Trans. Inform. Theory, 45(7):2495-2498, 1999.

[30] L.E. Danielsen and M.G. Parker. On the classification of all self-dual additive codes over GF(4) of length up to 12. arXiv:math.CO/0504522 2005.

[31] P. Delsarte. Bounds for unrestricted codes by linear programming. Philips Res. Reports, 27:272-289, 1972.

[32] C. Ding, D. Kohel, and S. Ling. Elementary 2-group character codes. IEEE Trans. Inform. Theory, 46(1):280-284, 2000.

[33] A. Ekert and C. Macchiavello. Error correction in quantum communication. Phys. Rev. Lett., $76: 2585-2588,1996$.

[34] K. Feng. Quantum codes $[[6,2,3]]_{p},[[7,3,3]]_{p}(p \geq 3)$ exist. IEEE Trans. Inform. Theory, 48(8):2384-2391, 2002.

[35] K. Feng. Quantum error-correcting codes. In Coding Theory and Cryptology, pages 91-142. World Scientific, 2002.

[36] K. Feng and Z. Ma. A finite Gilbert-Varshamov bound for pure stabilizer quantum codes. IEEE Trans. Inform. Theory, 50(12):3323-3325, 2004.

[37] M.H. Freedman and D.A. Meyer. Projective plane and planar quantum codes. Found. Comput. Math., 1(3):325-332, 2001.

[38] D. Gottesman. A class of quantum error-correcting codes saturating the quantum Hamming bound. Phys. Rev. A, 54:1862-1868, 1996.

[39] D. Gottesman. Pasting quantum codes. eprint: quant-ph/9607027 1996. 
[40] D. Gottesman. Stabilizer codes and quantum error correction. Caltech Ph. D. Thesis, eprint: quant-ph/9705052 1997.

[41] D. Gottesman. Fault-tolerant quantum computation with higher-dimensional systems. Chaos, Solitons, Fractals, 10(10):1749-1758, 1999.

[42] D. Gottesman. An introduction to quantum error correction. In S. J. Lomonaco, Jr., editor, Quantum Computation: A Grand Mathematical Challenge for the Twenty-First Century and the Millennium, pages 221-235, Rhode Island, 2002. American Mathematical Society. eprint: quant-ph/0004072

[43] D. Gottesman. Quantum error correction and fault-tolerance. eprint: quant-ph/0507174 2005.

[44] M. Grassl. Algorithmic aspects of error-correcting codes. In R. Brylinski and G. Chen, editors, The Mathematics of Quantum Computing, pages 223-252. CRC Press, 2001.

[45] M. Grassl and T. Beth. Quantum BCH codes. In Proc. X. Int'l. Symp. Theoretical Electrical Engineering, Magdeburg, pages 207-212, 1999.

[46] M. Grassl and T. Beth. Cyclic quantum error-correcting codes and quantum shift registers. Proc. Royal Soc. London Series A, 456(2003):2689-2706, 2000.

[47] M. Grassl, T. Beth, and M. Rötteler. On optimal quantum codes. Internat. J. Quantum Information, 2(1):757-775, 2004.

[48] M. Grassl, W. Geiselmann, and T. Beth. Quantum Reed-Solomon codes. In Applied algebra, algebraic algorithms and error-correcting codes (Honolulu, HI, 1999), volume 1719 of Lecture Notes in Comput. Sci., pages 231-244. Springer, Berlin, 1999.

[49] M. Grassl, M. Rötteler, and T. Beth. Efficient quantum circuits for non-qubit quantum error-correcting codes. Internat. J. Found. Comput. Sci., 14(5):757-775, 2003.

[50] L.C. Grove. Classical Groups and Geometric Algebra. Graduate Studies in Mathematics. American Mathematical Society, 2001.

[51] T. Hiramatsu and G. Köhler. Coding Theory and Number Theory. Kluwer Academic Publishers, London, 2003.

[52] W. C. Huffman and V. Pless. Fundamentals of Error-Correcting Codes. University Press, Cambridge, 2003.

[53] T. Kasami, S. Lin, and W. W. Peterson. New generalizations of the Reed-Muller codes Part I : Primitive codes. IEEE Trans. Inform. Theory, 14(2):189-199, 1968.

[54] J.-L. Kim. New quantum-error-correcting codes from Hermitian self-orthogonal codes over GF(4). In Proc. of the Sixth Intl. Conference on Finite Fields and Applications, Oaxaca, Mexico, May 21-25, pages 209-213. Springer-Verlag, 2002.

[55] J.-L. Kim and V. Pless. Designs in additive codes over GF(4). Designs, Codes and Cryptography, 30:187-199, 2003.

[56] J.-L. Kim and J. Walker. Nonbinary quantum error-correcting codes from algebraic curves. submitted to a special issue of $\mathrm{Com}^{2} \mathrm{MaC}$ Conference on Association Schemes, Codes and Designs in Discrete Math, 2004.

[57] A.Y. Kitaev. Quantum computations: algorithms and error correction. Russian Math. Surveys, 52(6):1191-1249, 1997. 
[58] A. Klappenecker and M. Rötteler. Beyond stabilizer codes II: Clifford codes. IEEE Transaction on Information Theory, 48(8):2396-2399, 2002.

[59] E. Knill. Group representations, error bases and quantum codes. Los Alamos National Laboratory Report LAUR-96-2807, 1996.

[60] E. Knill. Non-binary unitary error bases and quantum codes. Los Alamos National Laboratory Report LAUR-96-2717, 1996.

[61] E. Knill and R. Laflamme. A theory of quantum error-correcting codes. Physical Review A, 55(2):900-911, 1997.

[62] G. Lachaud and J. Wolfmann. The weights of the orthogonals of the extended quadratic binary Goppa codes. IEEE Trans. Inform. Theory, 36(3):686-692, 1990.

[63] V.I. Levenshtein. Krawtchouk polynomials and universal bounds for codes and designs in Hamming spaces. IEEE Trans. Inform. Theory, 41(5):1303-1321, 1995.

[64] R. Li and X. Li. Binary construction of quantum codes of minimum distance three and four. IEEE Trans. Inform. Theory, 50(6):1331-1336, 2004.

[65] D. J. C. MacKay, G. Mitchison, and P. L. McFadden. Sparse-graph codes for quantum error correction. IEEE Trans. Inform. Theory, 50(10):2315-2330, 2004.

[66] F.J. MacWilliams. A theorem on the distribution of weights in a systematic code. Bell Syst. Tech. J., 42:79-94, 1963.

[67] F.J. MacWilliams and N.J.A. Sloane. The Theory of Error-Correcting Codes. North-Holland, 1977 .

[68] W.J. Martin. A physics-free introduction to quantum error correcting codes. Util. Math., pages 133-158, 2004.

[69] R. Matsumoto. Improvement of Ashikhmin-Litsyn-Tsfasman bound for quantum codes. IEEE Trans. Inform. Theory, 48(7):2122-2124, 2002.

[70] R. Matsumoto and T. Uyematsu. Constructing quantum error correcting codes for $p^{m}$-state systems from classical error correcting codes. IEICE Trans. Fundamentals, E83-A(10):1878$1883,2000$.

[71] R.J. McEliece, E.R. Rodemich, jr. H. Rumsey, and L.R. Welch. New upper bounds on the rate of a code via the Delsarte-MacWilliams inequalities. IEEE Trans. Inform. Theory, 23(2):157, 1977.

[72] O. Moreno and C. J. Moreno. The Macwilliams-Sloane conjecture on the tightness of the Carlitz-Uchiyama bound and the weights of the duals of BCH codes. IEEE Trans. Inform. Theory, 40(2):1894-1907, 1994.

[73] O. Moreno, J. P. Pederson, and D. Polemi. An improved Serre bound for elementary abelian extensions of $\mathbf{F}_{q}(x)$ and the generalized Hamming weights of duals of $\mathrm{BCH}$ codes. IEEE Trans. Inform. Theory, 44(3):1291-1293, 1998.

[74] O. Ore. Galois connexions. Trans. Am. Math. Soc., 55:493-513, 1944.

[75] R. Pellikaan and X.-W. Wu. List decoding of $q$-ary Reed-Muller codes. IEEE Trans. Inform. Theory, 50(4):679-682, 2004. 
[76] M. S. Postol. A proposed quantum low density parity check code. eprint: quant-ph/0108131 2001.

[77] E.M. Rains. Quantum weight enumerators. IEEE Trans. Inform. Theory, 44(4):1388-1394, 1998.

[78] E.M. Rains. Monotonicity of the quantum linear programming bound. IEEE Trans. Inform. Theory, 45(7):2489-2492, 1999.

[79] E.M. Rains. Nonbinary quantum codes. IEEE Trans. Inform. Theory, 45:1827-1832, 1999.

[80] E.M. Rains. Quantum codes of minimum distance two. IEEE Trans. Inform. Theory, 45(1):266-271, 1999.

[81] E.M. Rains. Quantum shadow enumerators. IEEE Trans. Inform. Theory, 45(7):2361-2366, 1999

[82] E.M. Rains. Polynomial invariants of quantum codes. IEEE Trans. Inform. Theory, 46(1):5459,2000 .

[83] M. Rötteler, M. Grassl, and T. Beth. On quantum MDS codes. In Proc. 2004 IEEE Intl. Symposium on Information Theory, Chicago, USA, page 355, 2004.

[84] P. K. Sarvepalli and A. Klappenecker. Nonbinary quantum Reed-Muller codes. In IEEE International Symposium on Information Theory, 2005.

[85] D. Schlingemann. Stabilizer codes can be realized as graph codes. Quantum Inf. Comput., 2(4):307-323, 2002.

[86] D. Schlingemann and R.F. Werner. Quantum error-correcting codes associated with graphs. eprint: quant-ph/0001211 l, 2000.

[87] R. Schoof. Families of curves and weight distributions of codes. Bulletin Amer. Mathematical Society, 32(2):171-183, 1995.

[88] R. Schoof, G. van der Geer, and M. van der Vlugt. Weight formulas for ternary Melas codes. Math. Comp., 58:781-792, 1992.

[89] P. Shor. Scheme for reducing decoherence in quantum memory. Phys. Rev. A, 2:2493-2496, 1995.

[90] P. Shor and R. Laflamme. Quantum analog of the MacWilliams identities in classical coding theory. Phys. Rev. Lett., 78:1600-1603, 1997.

[91] A. Steane. Quantum Reed-Muller codes. IEEE Trans. Inform. Theory, 45(5):1701-1703, 1999.

[92] A.M. Steane. Multiple-particle interference and quantum error correction. Proc. Roy. Soc. London A, 452:2551-2577, 1996.

[93] A.M. Steane. Simple quantum error correcting codes. Phys. Rev. Lett., 77:793-797, 1996.

[94] A.M. Steane. Enlargement of Calderbank-Shor-Steane quantum codes. IEEE Trans. Inform. Theory, 45(7):2492-2495, 1999.

[95] H. Stichtenoth and C. Voß. Generalized Hamming weights of trace codes. IEEE Trans. Inform. Theory, 40(2):554-558, 1994. 
[96] A. Thangaraj and S.W. McLaughlin. Quantum codes from cyclic codes over $\operatorname{GF}\left(4^{m}\right)$. IEEE Trans. Inform. Theory, 47(3):1176-1178, 2001.

[97] G. van der Geer and M. van der Vlugt. Generalized Hamming weights of Melas codes and dual Melas codes. SIAM J. Disc. Mathematics, 7(4):554-559, 1980.

[98] F. Vatan, V.P. Roychowdhury, and M.P. Anantram. Spatially correlated qubit errors and burst-correcting quantum codes. IEEE Trans. Inform. Theory, 45(5):1703-1708, 1999.

[99] L. Xiaoyan. Quantum cyclic and constacyclic codes. IEEE Trans. Inform. Theory, 50(3):547$549,2004$. 